\title{
Article \\ Heteroaromatic N-Oxides Modified with a Chiral Oxazoline Moiety, Synthesis and Catalytic Applications
}

\author{
Zuzanna Wrzeszcz (D) and Renata Siedlecka *(D) \\ Department of Organic and Medicinal Chemistry, Faculty of Chemistry, Wroclaw University of Science and \\ Technology, Wyb. Wyspianskiego 27, 50-370 Wroclaw, Poland; zuzanna.wrzeszcz@pwr.edu.pl \\ * Correspondence: renata.siedlecka@pwr.edu.pl; Tel.: +48-71-3203224
}

\begin{abstract}
Interesting properties of N-oxides and pyridine oxazoline compounds have become the starting point to synthesize compounds connecting both groups. A multi-step synthesis of a series of chiral oxazoline substituted pyridine $\mathrm{N}$-oxides, alkyl derived of pyridine $\mathrm{N}$-oxides, bipyridine $\mathrm{N}$-oxides, and isoquinoline $\mathrm{N}$-oxides, based on amino alcohols derived from natural amino acids or other previously prepared, is presented herein. Various synthetic pathways have been designed and tested according to the properties and limitations imposed by the target products. The encountered problems related to the stability of the products were discussed. The resulting compounds (eighteen structures) were tested as catalysts in th e allylation of benzaldehyde (obtaining up to $79 \%$ ee) as well as in nitroaldol reaction (obtaining up to $48 \%$ ee).
\end{abstract}

Keywords: chiral heteroaromatic N-oxides; oxazolines; organo-catalysis; asymmetric transformations

Citation: Wrzeszcz, Z.; Siedlecka, R. Heteroaromatic N-Oxides Modified with a Chiral Oxazoline Moiety, Synthesis and Catalytic Applications. Catalysts 2021, 11, 444. https:// doi.org/10.3390/catal11040444

Academic Editors: Sylvain Oudeyer and Jean-François Brière

Received: 8 March 2021

Accepted: 26 March 2021

Published: 30 March 2021

Publisher's Note: MDPI stays neutral with regard to jurisdictional claims in published maps and institutional affiliations.

Copyright: (c) 2021 by the authors. Licensee MDPI, Basel, Switzerland. This article is an open access article distributed under the terms and conditions of the Creative Commons Attribution (CC BY) license (https:// creativecommons.org/licenses/by/ $4.0 /)$.

\section{Introduction}

An increasing interest in the development of the chemistry and applications of Noxides has been observed in the last decade. Apart from the fact that azaaromatic N-oxides can be found in nature [1] and their biological activity is recognized, due to their unique properties being a consequence of the $\mathrm{N}-\mathrm{O}$ moiety presence, they have found various applications. They can serve as oxidants [2], intermediates having significant synthetic value en route to diversely functionalized N-heterocycles, and in industrial syntheses of pharmaceuticals [3]. N-oxides are key components for ubiquitously used materials such as detergents, toilet soaps, toothpaste, shampoos, and cosmetics [4]. N-oxidation dramatically alters the reactivity of the parent base by activating the heteroaromatic ring towards both nucleophilic and electrophilic reagents [5].

Heteroaromatic N-oxides are also applied as catalysts in both metal-free transformations and metal-catalyzed reactions [6]. The great versatility of aromatic N-oxides is caused by their dual nature. As mild Lewis bases with basicity lower than corresponding pyridines, but sufficient enough to catalyze a wide variety of reactions and to activate Lewis acids, e.g., the C-Si bond in halo-silane compounds, they are able to increase Lewis acids nucleophilicity towards various reactions with electrophiles and allow them to react under conditions in which normally they would not be able to [1]. On the other hand, powerful electron-pair, N-oxide donors can act as ligands for transition metal complexes [6-8]. The important advantage of these ligands is that their synthesis is considerably facilitated by the rich chemistry of heteroaromatic N-oxides. However, catalytically efficient heteroaromatic $\mathrm{N}$-oxides are usually quite complex, sophisticated structures that require long and tedious synthesis. It seems justified to constantly search for relatively simple, easily available catalysts with an analogous catalytic potential. On the other side, azoles, specifically the 2-oxazoline sub-class of azoles, represent an important group of chiral auxiliaries and metal-binding agents that are used extensively in both enantio-selective catalysis and materials science [9-14]. 
The great majority of oxazolines are derived from readily accessible chiral amino alcohols, transformed in few high-yielded steps. The specific structure of the oxazoline ring makes this moiety a perfect chiral coordinating center, hence it has a direct influence on the stereochemical outcome of the reaction. In fact, oxazolines are widely applicable in metal-catalyzed transformations and they have become one of the most effective, versatile, and widely used classes of ligands for asymmetric catalysis $[10,15]$. Pyridineoxazoline-type ligands have been known from 1986 when they were first designed by Brunner et al. [16], but only in recent years have the properties of such hybrid ligands been extensively reinvestigated [17]. Comprehensive studies resulting in high development of pyridine-oxazoline-type chiral compounds have led to several types of very effective ligands, such as Pybox, Box, Phox, and others, that have rapidly become popular for use in asymmetric catalysis and in several types of challenging asymmetric reactions [17]. This shows, besides their special properties, their great potential for application in asymmetric catalytic reactions. Despite the numerous uses of both N-oxides and oxazolines, chiral structures containing both active fragments have not been described. Within our investigations, several azaaromatic oxazolines, and their $\mathrm{N}$-oxides based on frameworks of pyridine (4), alkyl-substituted pyridine (10 and 15), 2,2'-bipyridine (5), and isoquinoline (20) were synthesized with the aim of combining the unique properties of both moieties.

\section{Results and Discussion}

In general, heteroaromatic N-oxides have been easily obtained via oxidation of heterocycles. The procedure for the methyltrioxorhenium (MTO) mediated oxidation of pyridines was developed by Sharpless [18], but despite its simplicity and efficiency it has several limitations. Procedures using $m$-chloro-peroxy-benzoic acid ( $m$-CPBA) in mild conditions seem to be more universal [19]. There are two general approaches to obtain an oxidized nitrogen atom in the designed bifunctional compounds, shown in Scheme 1. The first assumes oxidation before oxazoline ring closure (A), while the second consists of the oxidation of previously received heteroaromatic-oxazoline-type compounds (B). The introduction of an oxazoline ring into the molecule can be accomplished by reacting the chiral amino alcohol with an oxidized or non-oxidized aromatic nitrile or carboximidate.

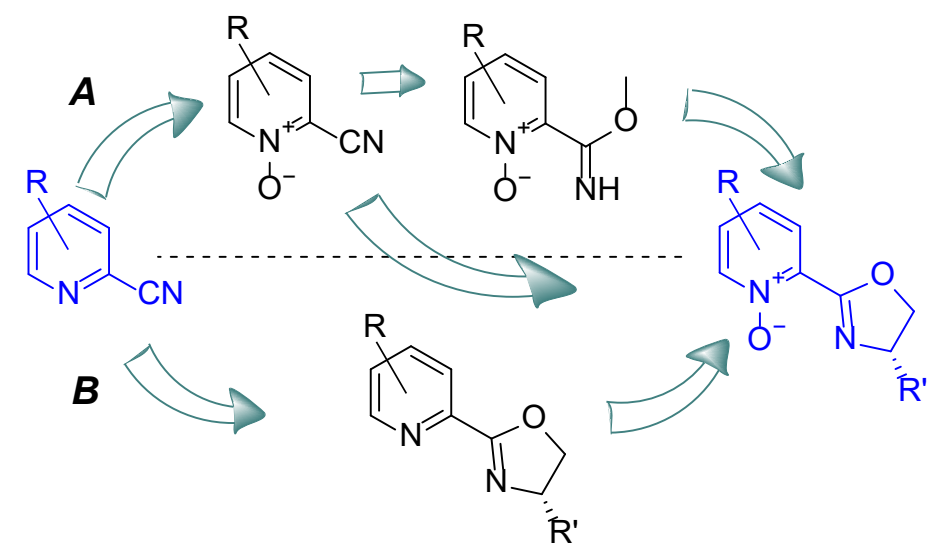

Scheme 1. Possible routes from heteroaromatic cyanides to chiral oxazoline-derived azaaromatic N-oxides.

\subsection{Pyridine N-Oxide Derivatives Preparation}

The first attempts involved oxidation, before introducing an oxazoline ring into the structure. The oxidation of azaaromatic nitriles applying the $\mathrm{H}_{2} \mathrm{O}_{2}$ / MTO system [18] was followed by transformation to carboximidate with sodium methanolate [20,21], and then, following the literature data, the reaction between 2-pyridine $\mathrm{N}$-oxide imidate and amino alcohol catalyzed with $\mathrm{ZnCl}_{2}$ in refluxed chlorobenzene [22]. Unfortunately, in the postreaction mixture, both the oxidized and deoxidized form of the expected oxazoline were observed, which suggests that too harsh reaction conditions were applied. Aromatic N- 
oxides are relatively stable and resistant to deoxygenation. Conventional methods for the deoxygenation of N-oxides involve the use of trivalent phosphorous compounds [23-25] or the application of excessive metals such as iron or zinc [26]. A range of catalytic procedures have also been developed applying transition metals in combination with hydrogen gas [27], or by using rhenium, molybdenum, or palladium catalysts with triphenylphosphine or triethylamine [28-30].

However, deoxidation of the heteroaromatic N-oxides in mild conditions, similar to those observed in our experiments, was recently reported in the case of the reaction involving the presence of Lewis acid (namely $\mathrm{ZnBr}_{2}$ ) [31].

The alternative approach, involving the reaction of 2-pyridine N-oxide imidate with amino alcohol in the presence of molecular sieves in toluene at elevated temperature, previously reported as effective in the preparation of appropriate pyridine-oxazoline derivatives [32], has been introduced. However, after five days of reaction, the unreacted substrates were still observed; moreover, the purification by acidic extraction or column chromatography led to partial hydrolysis of the product. Hence, it was necessary to conduct a reaction for a time that provided a complete conversion of substrates. The optimum was achieved after seven days of the reaction. For the preparation of chiral oxazoline derivatives, different commercially available amino alcohols were used, as well as the amino alcohol obtained from camphor quinone according to the procedure described in the literature [33]. The nine novel chiral oxazoline $\mathrm{N}$-oxides-five with pyridine $\mathrm{N}$-oxide fragment (4a-e) and the four with 2,2'-bipyridine $\mathrm{N}$-oxide fragment $(\mathbf{5 a} \mathbf{a}-\mathbf{d})$ - -have been obtained using this procedure, with generally high overall yields (Scheme 2). When $2-$ bipyridyl cyanide was applied as a substrate, oxidation took place in the unsubstituted pyridine ring, according to previous literature reports [25] (observation confirmed by NMR correlation spectra). Therefore, the finally obtained chiral $\mathrm{N}$-oxide-oxazoline derivatives (5a-d) have a slightly different structure than respective $\mathrm{N}$-oxide-pyridine derivatives, and the oxazoline ring was at a long-distance from the $\mathrm{N}$-oxide moiety.

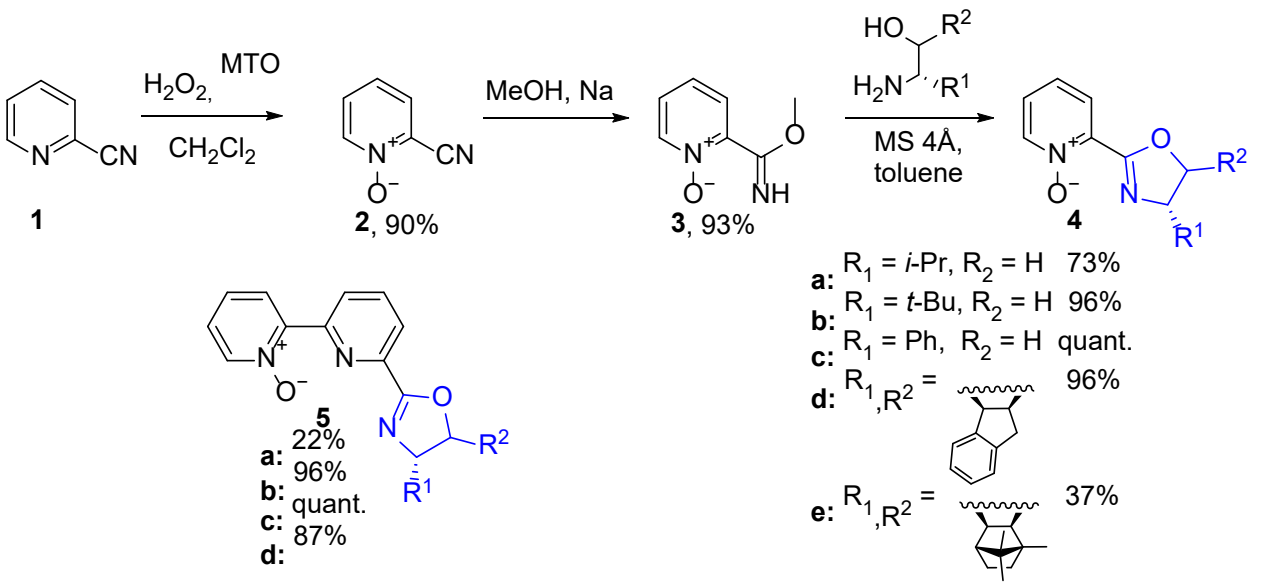

Scheme 2. Synthesis of 2-pyridine N-oxide-derived oxazolines.

Oxazolines containing azaaromatic $\mathrm{N}$-oxides tended to be not very stable. In simple tests, it was confirmed that the obtained chiral oxazoline $\mathrm{N}$-oxides are unstable, sensitive to moisture and other factors. They decomposed/hydrolyzed to opened hydroxy amides during preparative chromatography or when not stored under neutral conditions. The respective amido-alcohol formed by opening the oxazoline ring was identified as the transformation product.

The hydrolysis process may presumably involve the protonation of the oxazoline ring nitrogen and a nucleophile ring-opening attack, as is shown in Scheme 3, similar to the ring-opening polymerization initiation step reported in the literature [34]. 
<smiles></smiles>

Scheme 3. Schematic representation of the possible oxazoline ring opening.

\subsection{3,5-Lutidine N-oxide Derivatives Preparation}

Transformation of 2-cyano-3,5-lutidine into respective imidate provided caused some problems. The reaction of 2-cyano-3,5-dimethylpyridine (8) did not occur at all, while the reaction of corresponding N-oxide (9) proceeded with a very low yield of about $13 \%$; moreover, even a three-fold increase of solution concentration did not affect efficiency. An attempt consisting of the direct reaction between 2-cyano-3,5-lutidine (8) and chiral amino alcohol in the presence of $\mathrm{Zn}(\mathrm{OTf})_{2}$ as a catalyst, followed by the oxidation of received oxazoline with $m$-CPBA $(70 \%)$, gave a low yield, e.g., only $10 \%$ of the overall yield resulted in the case of $(1 R, 2 S)-(+)$-cis-1-amino-2-indanol. More effective was the direct oxazoline ring closure reaction of previously obtained 2-cyano-3,5-lutidine $\mathrm{N}$-oxide (9) with the chiral amino alcohols. Finally, lutidine-oxazoline N-oxide derivatives (10a-d) were obtained in low to moderate yields (19-49\%, see Scheme 4 ).

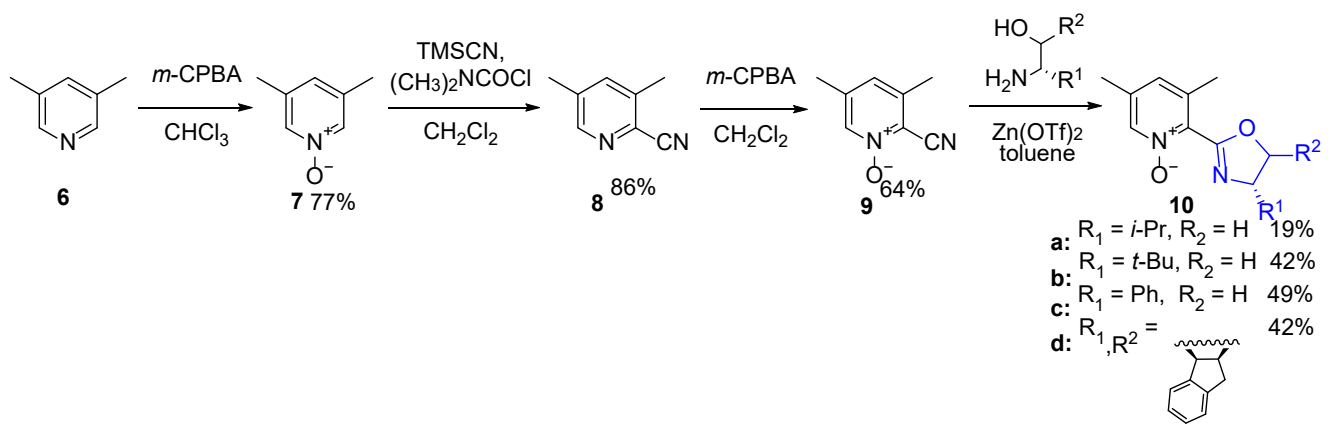

Scheme 4. Synthesis of 2-(3,5-lutidine) N-oxide-derived oxazolines.

\subsection{2-tert-Butylpyridine N-Oxide Derivatives Preparation}

Another procedure turned out to be necessary for the 2-tert-butylpyridine derivative. Its 6-carbonitrile (13) proved to be resistant to oxidation with common oxidants such as $\mathrm{H}_{2} \mathrm{O}_{2}$ or $m$-CPBA, but the formation of the corresponding carboximidate (with sodium methanolate) was very efficient ( $94 \%$ yield). This fact opened the path for the synthesis of oxazoline-derivatives via ring closure in the presence of molecular sieves, which was efficient in the preparation of pyridine and bipyridine oxazolines (see Scheme 5). Novel chiral 6-tert-butylpyridine oxazolines (15c, 15d) with good overall yield were obtained. The same strategy was highly efficient also in preparing previously reported derivatives (15a and 15b, [22,35]); however, their oxidation failed. However, considering their moisture sensitivity, 100\% m-CPBA was used for the oxidation reaction. Despite the precautions taken, in this case too opening of the oxazoline ring took place and the corresponding hydroxy amides were isolated as the only products $(\mathbf{1 6 a}-\mathbf{e})$.

\subsection{Isoquinoline N-Oxide Derivatives Preparation}

The procedure applied for the N-oxide of 2-cyanopyridine-oxazoline derivatives synthesis was not useful in the case of the respective isoquinoline derivatives preparation. The solubility of 1-isoquinolinecarbonitrile N-oxide (19) in methanol was limited, and preparation of the respective imidate in dry toluene did not bring the expected improvement. The procedure based on the direct formation of oxazolines from 1-isoquinolinecarbonitrile with a catalytic amount of zinc triflate [36], followed by the oxidation of obtained N-heteroaromatic oxazolines (19), seemed more promising (Scheme 6) [37,38]. However, reaction with the 
(S)-phenyl-glycinol gave an unexpected result. Attachment of two amino alcohol molecules (21) was the major product (up to $50 \%$ ), isolated each time irrespective of the catalyst amount used $(15,5$, or $1 \mathrm{~mol} \%)$. Since the formation of hydrolysis product next to the desired oxazoline was observed in the reaction mixture, it may be suspected that product 21 was formed via the opening of the latter, due to the nucleophilic attack of phenyl-glycinol nitrogen on the positively polarized carbon atom in 19c. The application of zinc chloride resulted in the formation of the same structure. The changing of order, and earlier oxidation, followed by the reaction of 1-isoquinoline carbonitrile N-oxide with $\mathrm{Zn}(\mathrm{OTf})_{2}$, allowed us to receive the desired product (20c), yielding $58 \%$ (Scheme 6). In the same reaction the previously prepared $(1 R, 2 S, 3 R, 4 S)$-(-)-3-amino-1,7,7-trimethylbicyclo[2.2.1] hep-tan-2-ol was applied, giving the $\mathrm{N}$-oxide $20 \mathrm{e}$, but in a low yield $(10 \%)$.

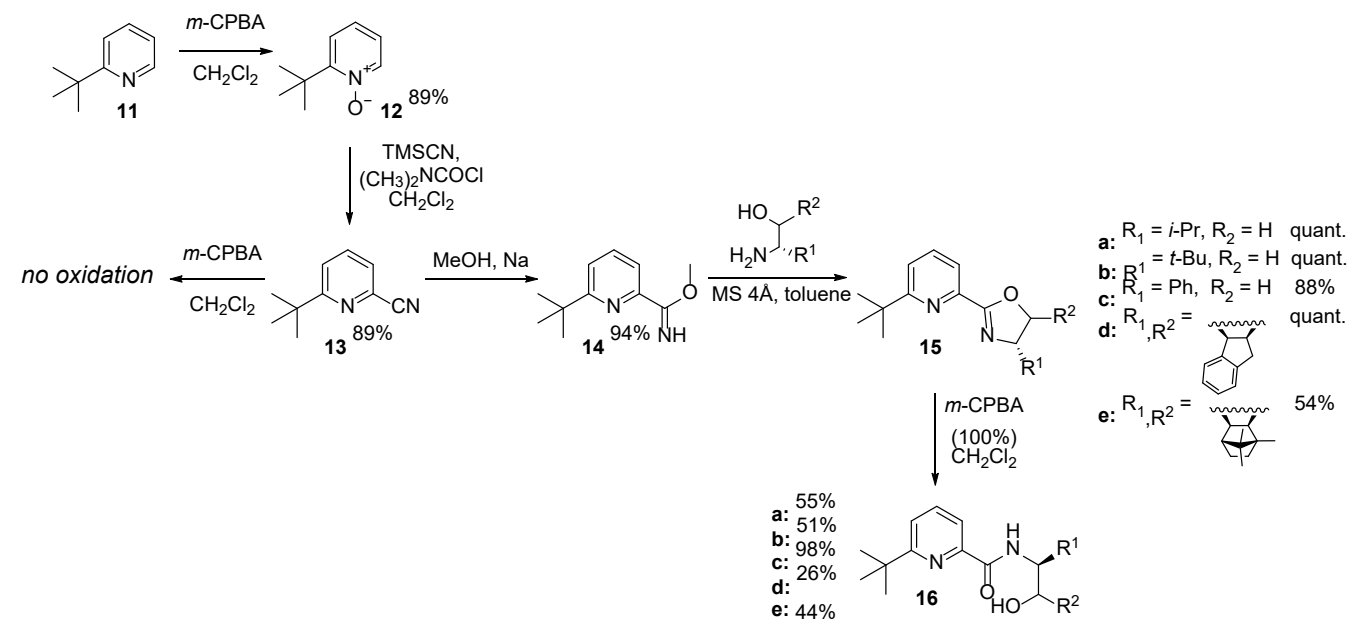

Scheme 5. Synthesis of 2-(6-tert-butylpyridine) N-oxide-derived oxazolines.

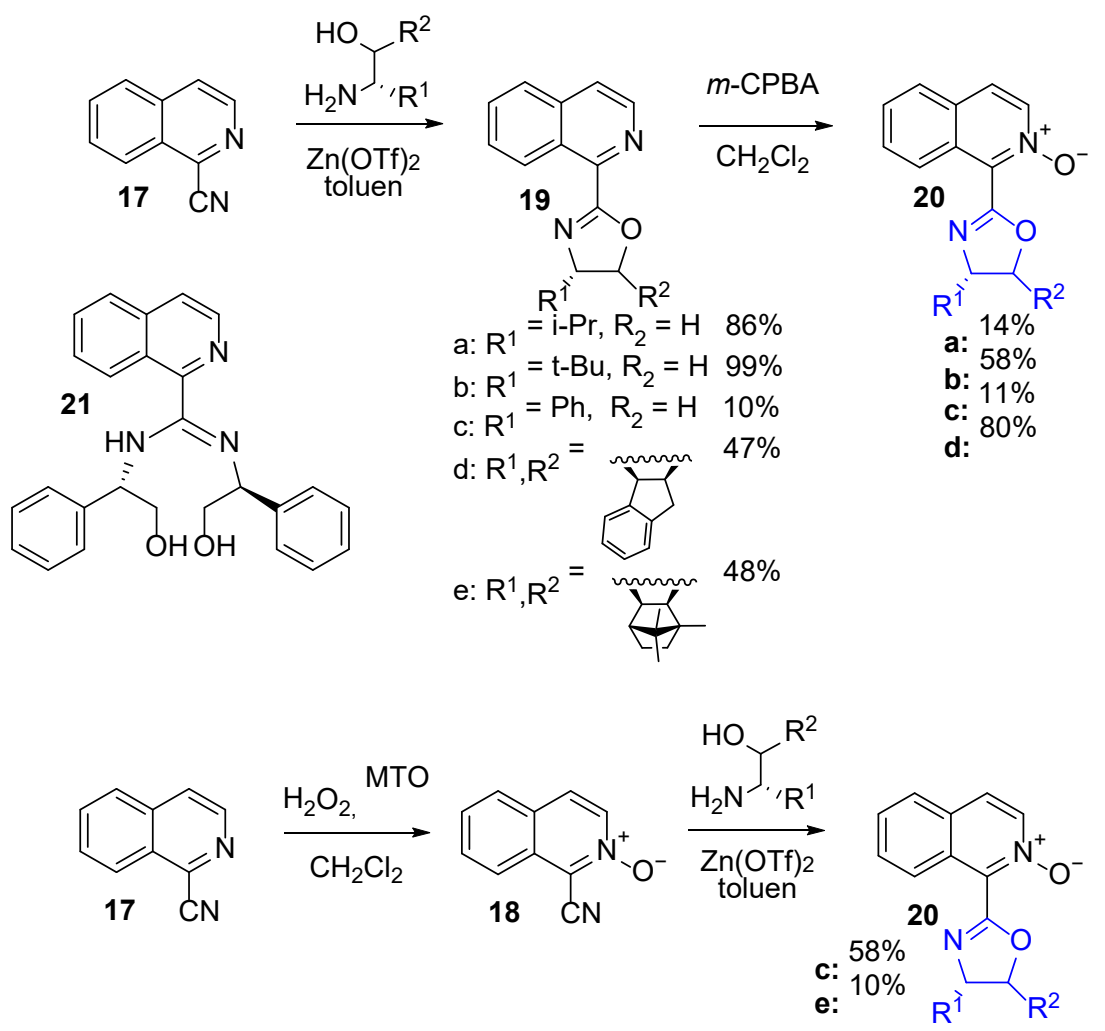

Scheme 6. Synthesis of 1-isoquinoline N-oxide-derived oxazolines. 


\subsection{2-Pyridine-imidazole-N-oxide Preparation}

Having in hand heteroaromatic nitriles, and inspired by Tydlitát et al. [20], we decided to obtain $\mathrm{NH}$-imidazolines with $\mathrm{N}$-oxide fragment and with $(1 R, 2 R)$-diamino-cyclo-hexane $(\mathrm{DACH})$ as a source of chirality. Normally the two-step introduction reaction of a hexahydro-benzimidazole group in place of $\mathrm{CN}$ group can be conducted as a single-step reaction of DACH with in situ generated carboximidate transition product, without the necessity of isolation. We found that the only sequence successfully leading to the desired product was the oxidation of the 2-pyridine nitrile and then the introduction of the imidazole function (Scheme 7). Following this procedure, 2-pyridine-imidazole derivative 22 and 2-bipyridineimidazole derivative $\mathbf{2 6}$ were obtained, respectively. Attempts to oxidize the previously synthesized pyridine-imidazole derivative were either unsuccessful (with $\mathrm{MTO} / \mathrm{H}_{2} \mathrm{O}_{2}$ ) or resulted in aromatization of the five-membered imidazole ring (with $m$-CPBA) and loss of chirality.

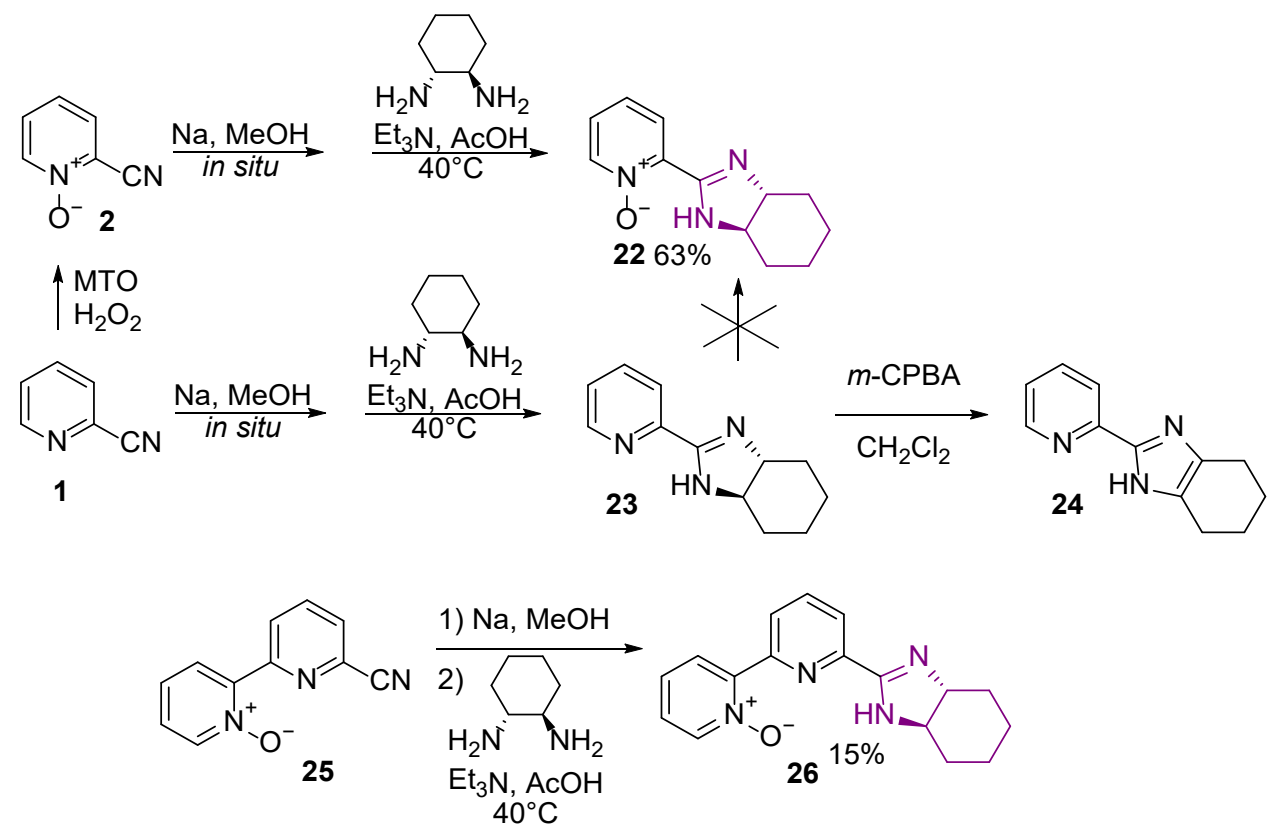

Scheme 7. Synthesis of 2-pyridine-imidazole-N-oxides.

\subsection{Catalytic Applications}

Using the ability of the $\mathrm{N}$-oxide moiety to activate the silane compound and an additional coordination place in the oxazoline or imidazole ring, we decided to examine selected compounds in the allylation of benzaldehyde with allyl-trichlorosilane which has become the testing ground for catalytic properties of new chiral Lewis bases. Our typical procedure involved the use of $1.5 \%$ mol of the catalyst, 1.175 equivalents of allyl-trichlorosilane, and 5 equivalents of diisopropylethylamine (DIPEA) in acetonitrile at $-40^{\circ} \mathrm{C}$. The yields of isolated products and enantiomeric excesses, determined by high performance liquid chromatography (HPLC), are collected in Table 1. Generally, the (S) absolute configuration was assigned to the predominant isomer of homoallylic alcohol formed, by comparison of retention time values with the literature [39]. Creation of allyl alcohol with $(R)$ absolute configuration was observed when the appropriate $(1 R, 2 S)$-(+)-cis-1-amino-2-indanol derivatives $(4 \mathrm{~d}, 5 \mathbf{d}, 20 \mathrm{~d})$ and $(1 R, 2 R)$-DACH derivative (22) were used as a source of chirality. Compounds based on bipyridine $\mathrm{N}$-oxide (5a-d) gave mediocre results, possibly due to the large distance between the N-oxide fragment and the chiral center, and because of the possibility of free rotation along the bond linking the heteroaromatic rings. The low ee value obtained with the imidazoline-based pyridine $\mathrm{N}$-oxide (22) may be due to the supposed formation of a hydrogen bond between the $\mathrm{NH}$ of the imidazoline ring and the oxygen of the N-oxide group (see Figure 1), resulting in its deactivation for the silane compound. 
Table 1. Allylation of benzaldehyde with allyl-trichlorosilane catalyzed by obtained N-oxides a .

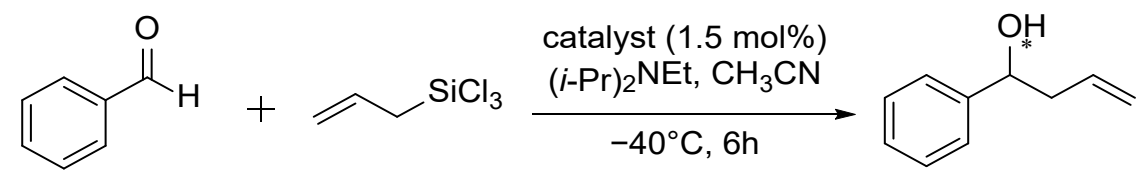

\begin{tabular}{|c|c|c|c|c|}
\hline Entry & Catalyst & Yield $(\%)^{b}$ & ee $(\%)^{c}$ & Configuration ${ }^{d}$ \\
\hline 1 & $4 a$ & 61 & 18 & $S$ \\
\hline 2 & $4 b$ & 20 & 26 & $S$ \\
\hline 3 & $4 c$ & 21 & 71 & $S$ \\
\hline $4^{\mathrm{e}}$ & $4 c$ & 26 & 62 & $S^{f}$ \\
\hline 5 & $4 d$ & 28 & 23 & $R^{f}$ \\
\hline 6 & $4 e$ & 90 & $<5$ & - \\
\hline 7 & $5 a$ & 0 & - & - \\
\hline 8 & $5 b$ & 12 & 41 & $S$ \\
\hline 9 & $5 c$ & 0 & - & - \\
\hline 10 & $5 d$ & $<10$ & 23 & $R$ \\
\hline 11 & $10 \mathrm{a}$ & 18 & 19 & $S^{f}$ \\
\hline 12 & $10 \mathrm{~b}$ & $<10$ & 27 & $S$ \\
\hline 13 & $10 \mathrm{c}$ & 35 & 79 & $S^{f}$ \\
\hline 14 & $10 \mathrm{~d}$ & 18 & 8 & $S$ \\
\hline 15 & $20 a$ & 13 & $<10$ & $S$ \\
\hline 16 & $20 \mathrm{~b}$ & 0 & - & - \\
\hline 17 & $20 c$ & 27 & 57 & $S$ \\
\hline 18 & $20 \mathrm{~d}$ & $<10$ & 24 & $R$ \\
\hline 19 & $20 \mathrm{e}$ & 49 & $<10$ & - \\
\hline 20 & 22 & 39 & 36 & $R^{f}$ \\
\hline
\end{tabular}

a The reaction was carried out at a $0.6 \mathrm{mmol}$ scale in $\mathrm{CH}_{3} \mathrm{CN}(3 \mathrm{~mL})$ with allyl-trichlorosilane (1.175 eq.) and diisopropylethylamine (DIPEA) (5 eq.). ${ }^{\mathrm{b}}$ Isolated yields after flash chromatography. ${ }^{\mathrm{c}}$ Determined by high performance liquid chromatography (HPLC) on a Chiralpak AS-H column ( $n$-hexane: $i / \mathrm{PrOH}$ 99:1; $0.6 \mathrm{~mL} / \mathrm{min}$; $\lambda 220 \mathrm{~nm}: \mathrm{t}_{\mathrm{R}}=30.3 \mathrm{~min}, \mathrm{t}_{\mathrm{S}}=33.4 \mathrm{~min}$ (see Figures S31-S47). ${ }^{\mathrm{d}}$ Assigned by comparison of the retention times.

e Reaction run in $\mathrm{CH}_{2} \mathrm{Cl}_{2} .{ }^{\mathrm{f}}$ Measured optical rotation given in Supplementary Materials.

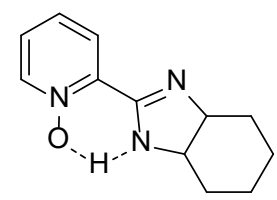

Figure 1. Representation of the possible hydrogen bond formation in $\mathbf{2 2 .}$

Better results were achieved for pyridine N-oxide oxazolines 4 (12-90\% yield, 19-71\% ee). For all, i.e., pyridine N-oxide, 3,5-lutidine N-oxide, and isoquinoline N-oxides oxazolines, the same tendency was observed. The highest enantioselectivity was observed when the catalyst with (S)-phenyl-glycinol fragment was applied (71\% ee for $4 \mathbf{c}$, $79 \%$ ee for $\mathbf{1 0 c}$, and $57 \%$ for 20c). Changing the solvent to dichloromethane (for 4c) did not improve the results. The obtained yield was slightly higher (from $21 \%$ to $26 \%$ ) while the enantioselectivity decreased (from $71 \%$ to $62 \%$ ). A control experiment using 2,2'-bipyridyl N-oxide as a catalyst gave the allylation product with a yield of $24 \%$, but racemic (see Figure S30), whereas, without the catalyst, reaction does not occur. The highest efficiency was obtained for the catalyst with a camphor-derived fragment, but with simultaneous racemization. These findings indicate that $\pi-\pi$ interactions might play an important role during the creation of the transition state, increasing the enantioselectivity, while the mono-terpenoid fragment with strong steric requirements enables high efficiency but not stereoselectivity. The assumed transition state options in the allylation reaction are shown in Figure 2a. One of these structures shows the possible parallel orientation of both aromatic rings, of the catalyst and the substrate. Such $\pi-\pi$ electronic interactions can additionally support the observed enantioselectivity. Similar effects have been discussed in the literature [40]. In the 
case of reactions with catalysts that do not allow such interactions, the obtained ee value is significantly lower.

(a)

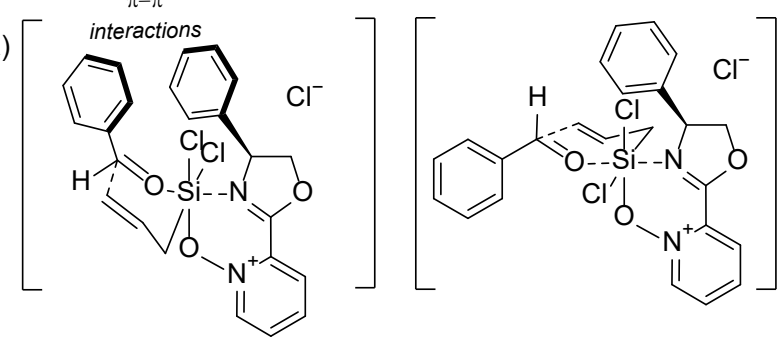

(b)

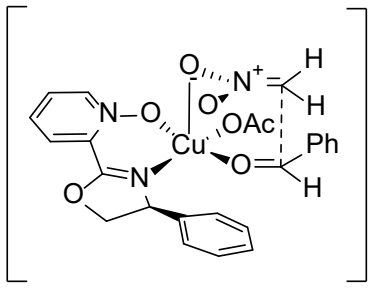

Figure 2. Representation of the possible transition state structures in allylation reaction (a) and nitroaldol reaction $(\mathbf{b})$.

The catalysts most effective for benzaldehyde were used in the allylation of p-nitrobenzoic and 1- and 2-naphthaldehydes. The results obtained were moderate (see Table 2), slightly higher yields were observed and the best enantioselectivity was achieved for 2-naphthaldehyde (59-72\% ee).

Table 2. Allylation of aromatic aldehydes with allyl-trichlorosilane catalyzed by selected N-oxides ${ }^{\text {a }}$.

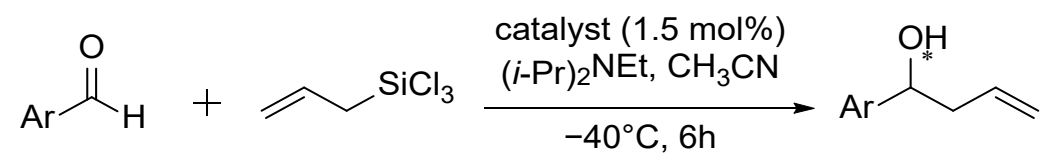

\begin{tabular}{|c|c|c|c|c|c|}
\hline Entry & $\mathbf{R}$ & Catalyst & Yield (\%) ${ }^{b}$ & ee $(\%)^{c}$ & Configuration ${ }^{\mathrm{d}}$ \\
\hline 1 & $4-\mathrm{NO}_{2}-\mathrm{Ph}$ & $4 c$ & 54 & 15 & $S$ \\
\hline 2 & 1-naphthyl & $4 c$ & 59 & 47 & $S$ \\
\hline 3 & 2-naphthyl & $4 c$ & 60 & 72 & $S^{e}$ \\
\hline 4 & $4-\mathrm{NO}_{2}-\mathrm{Ph}$ & $10 \mathrm{c}$ & 52 & 31 & $S$ \\
\hline 5 & 1-naphthyl & $10 \mathrm{c}$ & 54 & 57 & $S$ \\
\hline 6 & 2-naphthyl & $10 \mathrm{c}$ & 46 & 68 & $S^{e}$ \\
\hline 9 & $4-\mathrm{NO}_{2}-\mathrm{Ph}$ & $20 c$ & 35 & 11 & $S$ \\
\hline 10 & 1-naphthyl & $20 \mathrm{c}$ & 24 & 47 & $S$ \\
\hline 11 & 2-naphthyl & $20 \mathrm{c}$ & 17 & 59 & $S$ \\
\hline
\end{tabular}

a The reaction was carried out on a $0.5 \mathrm{mmol}$ scale in $\mathrm{CH}_{3} \mathrm{CN}(2.5 \mathrm{~mL})$ with allyl-trichlorosilane (1.175 eq.) and DIPEA (5 eq.). ${ }^{\mathrm{b}}$ Isolated yields after flash chromatography. ${ }^{c}$ Determined by HPLC (see Figures S48-S56).

$\mathrm{d}$ Assigned by comparison of the retention times. ${ }^{\mathrm{e}}$ Measured optical rotation given in Supplementary Materials.

Using Pearson acid-base concept according to which pyridine nitrogen is a border-line base forming strong bonds with the same type of acids, e.g., copper (II) ion, we decided to examine selected compounds as ligands in the Henry reaction catalyzed by copper (II) complex. The main purpose of the conducted reactions was to test whether the pyridine $\mathrm{N}$-oxide oxazolines employed as ligands are able to give complexes that allow asymmetric induction. Therefore, the preparative isolation of the product was not performed and the yield was estimated based on the ${ }^{1} \mathrm{H}$ NMR spectrum. Reactions were carried out using $12 \% \mathrm{~mol}$ of ligand, $10 \% \mathrm{~mol}$ of $\mathrm{Cu}(\mathrm{OAc})_{2} \cdot \mathrm{H}_{2} \mathrm{O}$, and 10 equivalents of nitromethane in isopropanol at $0{ }^{\circ} \mathrm{C}$. Obtained results are collected in Table 3 . The applied ligands induced the same absolute configuration of nitro alcohols received as in the previous catalytic reaction. Generally, obtained ee values were lower, and not as scattered as in the case of the allylation reaction. Moreover, the application of pyridine N-oxide oxazoline with a camphor-derived fragment (4e) did not give a racemic mixture exclusively. It is visible that the high steric requirements of this ligand resulted in a higher selectivity in the nitroaldol reaction. The electronic effects in the transition state do not appear to be significant (see Figure 2b). 
Table 3. Nitroaldol reaction with benzaldehyde, catalyzed by selected N-oxides ${ }^{\text {a }}$.

\begin{tabular}{|c|c|c|c|c|c|}
\hline Entry & Ligand & Time (Days) & Yield (\%) b & ee $(\%)^{c}$ & Configuration $\mathrm{d}$ \\
\hline 1 & $4 a$ & 3 & 80 & 48 & $S$ \\
\hline 2 & $4 c$ & 6 & 60 & 37 & $S$ \\
\hline 3 & $4 \mathrm{e}$ & 6 & 85 & 28 & $R$ \\
\hline 4 & 22 & 6 & 68 & 35 & $R$ \\
\hline
\end{tabular}

a The reaction was carried out on a $0.5 \mathrm{mmol}$ scale in $i$-PrOH $(1 \mathrm{~mL})$ at $-0{ }^{\circ} \mathrm{C} .{ }^{\mathrm{b}}$ Yield calculated from ${ }^{1} \mathrm{H} \mathrm{NMR}$ spectrum. ${ }^{c}$ Determined by high performance liquid chromatography (HPLC) on a Chiracel OD- $\mathrm{H}, n$-hexane $/ i$ $\mathrm{PrOH}, 9: 1 ; 1.0 \mathrm{~mL} / \mathrm{min}, \lambda=225 \mathrm{~nm}: \mathrm{t}_{\mathrm{R}}=16.5 \mathrm{~min}, \mathrm{t}_{\mathrm{S}}=19.8 \mathrm{~min}$ (see Figures S57-S61). ${ }^{\mathrm{d}}$ Assigned by comparison of the retention times.

\section{Materials and Methods}

\subsection{General}

Solvents were distilled, and other reagents were used as received. Reactions were monitored by thin-layer chromatography (TLC) on silica gel 60 F-254 precoated plates (Merck, Darmstadt, Germany), and spots were visualized with a UV lamp. Products were purified by standard column chromatography on silica gel 60 (230-400 mesh) (Merck). Optical rotations at $578 \mathrm{~nm}$ were measured using an Optical Activity Ltd. (Huntington, UK) Model AA-5 automatic polarimeter. Melting points were determined using a Boëtius hotstage apparatus (PHMK VEB Analytic, Dresden, Germany). ${ }^{1} \mathrm{H}$ and ${ }^{13} \mathrm{C} \mathrm{NMR}(400 \mathrm{MHz}$ and $100 \mathrm{MHz}$, respectively) spectra were recorded in $\mathrm{CDCl}_{3}$ on Jeol 400yh and Bruker Avance II 600 instruments (Karlsruhe, Germany). High-resolution mass spectra (HRMS) were recorded using electrospray ionization mode on the Waters LCT Premier XE TOF spectrometer (Waters Corporation, Milford, MA, USA). The enantiomeric ratios of the samples were determined by chiral high-performance liquid chromatography (HPLC) measurements (Thermo Fisher Scientific, Waltham, MA, USA) using Chiracel OD-H, OB$\mathrm{H}$, or Chiralpak AS-H chiral columns. The configuration of the products was assigned by comparison to literature data. ${ }^{1} \mathrm{HNMR}$ and ${ }^{13} \mathrm{C}$ NMR spectra of all newly obtained compounds are shown in Supplementary Materials (Figure S1-S29).

\subsection{General Procedure for the Heteroaromates $\mathrm{N}$-Oxidation with $\mathrm{MTO} / \mathrm{H}_{2} \mathrm{O}_{2}$ System}

According to the Sharpless protocol [18], to a solution of carbonitrile $(9.6 \mathrm{mmol}$, 1 eq.) in dichloromethane $(3.8 \mathrm{~mL}), \mathrm{MTO}\left(5 \% \mathrm{~mol}\right.$ for 1,17 and $1 \%$ mol for $6^{\prime}$-cyano2,2'-bipyridine) and $30 \% \mathrm{H}_{2} \mathrm{O}_{2}(1.9 \mathrm{~mL}, 2$ eq.) was added. After $24 \mathrm{~h}$ (12 $\mathrm{d}$ in case of 25) of stirring at room temperature white precipitate could be seen and a biphasic reaction mixture was treated with a catalytic amount of $\mathrm{MnO}_{2}(15 \mathrm{mg})$ and stirred until oxygen evolution ceased. Following the addition of water, the water phase was extracted three times with $\mathrm{CH}_{2} \mathrm{Cl}_{2}(3 \times 5 \mathrm{~mL})$. The combined organic phases were dried over $\mathrm{Na}_{2} \mathrm{SO}_{4}$, filtered, and concentrated to give the desired compounds.

2-cyanopyridine 1-oxide (2), Yield: $1.04 \mathrm{~g}(90 \%)$; white needles; mp $122{ }^{\circ} \mathrm{C}$ (lit. [41] 119-120 ${ }^{\circ} \mathrm{C}$ ); following the protocol of Rassadin and Kukushkin [41], crude product can be purified by recrystallization from methanol to give 2-cyanopyridine 1 -oxide $(65 \%) ;{ }^{1} \mathrm{H} \mathrm{NMR}(400 \mathrm{MHz}$, DMSO- $\left.d_{6}\right): \delta=7.45(\mathrm{t}, \mathrm{J}=8 \mathrm{~Hz}, 1 \mathrm{H}), 7.71(\mathrm{t}, \mathrm{J}=8 \mathrm{~Hz}, 1 \mathrm{H}), 8.10(\mathrm{dd}, \mathrm{J}=8,4 \mathrm{~Hz}, 1 \mathrm{H}), 8.45(\mathrm{~d}$, $\mathrm{J}=8 \mathrm{~Hz}, 1 \mathrm{H})$; analytical data consistent with literature values [41].

6'-cyano-[2,2'-bipyridine] 1-oxide (25), Yield: $0.332 \mathrm{~g}, 86 \%$; white solid; mp 176-179 ${ }^{\circ} \mathrm{C}$; ${ }^{1} \mathrm{H} \mathrm{NMR}\left(400 \mathrm{MHz}, \mathrm{CDCl}_{3}\right): \delta=7.35(\mathrm{td}, \mathrm{J}=8,4 \mathrm{~Hz}, 1 \mathrm{H}), 7.43(\mathrm{t}, \mathrm{J}=8 \mathrm{~Hz}, 1 \mathrm{H}), 7.73(\mathrm{dd}$, $\mathrm{J}=8,1 \mathrm{~Hz}, 1 \mathrm{H}), 7.97(\mathrm{t}, \mathrm{J}=8 \mathrm{~Hz}, 1 \mathrm{H}), 8.25(\mathrm{dd}, \mathrm{J}=8,2 \mathrm{~Hz}, 1 \mathrm{H}), 8.34(\mathrm{~d}, \mathrm{~J}=8 \mathrm{~Hz}, 1 \mathrm{H})$, $9.20(\mathrm{dd}, \mathrm{J}=8,1 \mathrm{~Hz}, 1 \mathrm{H})$; analytical data consistent with literature values [42]. 
1-cyanoisoquinoline 2-oxide (18), Recrystallized from methanol; yield: $1.03 \mathrm{~g}, 93 \%$; pale yellow needles; mp 212-213 ${ }^{\circ} \mathrm{C}$ (lit. [43] $\left.207^{\circ} \mathrm{C}\right) ;{ }^{1} \mathrm{H}$ NMR $\left(400 \mathrm{MHz}, \mathrm{CDCl}_{3}\right): \delta=7.67(\mathrm{t}, \mathrm{J}=8 \mathrm{~Hz}$, $1 \mathrm{H}), 7.77-7.81(\mathrm{~m}, 2 \mathrm{H}), 7.85(\mathrm{~d}, \mathrm{~J}=8 \mathrm{~Hz}, 1 \mathrm{H}), 8.00(\mathrm{~d}, \mathrm{~J}=8 \mathrm{~Hz}, 1 \mathrm{H}), 8.16(\mathrm{~d}, \mathrm{~J}=8 \mathrm{~Hz}, 1 \mathrm{H})$.

3.3. General Procedure for the Preparation of Carboximidates-Pyridine, Bipyridine N-Oxide and Tert-Bu-Pyridine Carboximidates

Preparation of carboximidates was performed based on available literature procedures $[20,21]$ : the respective cyano $\mathrm{N}$-oxide $(4.7 \mathrm{mmol}, 1 \mathrm{eq}$.) was added to the prepared solution of sodium methoxide $(0.108 \mathrm{~g}, 1$ eq. of $\mathrm{Na}$ in anhydrous $\mathrm{MeOH}(20 \mathrm{~mL}))$. The mixture was stirred for $24 \mathrm{~h}$ at room temperature and then it was quenched by the stoichiometric additive of glacial acetic acid $(0.3 \mathrm{~mL}, 1$ eq.). After concentration in vacuo, the crude product was suspended in dichloromethane and filtered. The filtrate was evaporated affording the desired product.

2-(imino(methoxy)methyl)pyridine 1-oxide (3), Yield: $0.595 \mathrm{~g}$ (93\%); off-yellow solid; $\mathrm{mp} 107-110{ }^{\circ} \mathrm{C} .{ }^{1} \mathrm{H}$ NMR $\left(400 \mathrm{MHz}, \mathrm{CDCl}_{3}\right): \delta=3.91\left(\mathrm{~s}, 3 \mathrm{H}, \mathrm{CH}_{3}\right), 7.29-7.35(\mathrm{~m}, 2 \mathrm{H}$, Py), 7.99-8.02 (m 1H, Py), 8.27-8.29 (m, 1H, Py), 11.40 (br s, $1 \mathrm{H}, \mathrm{NH}) ;{ }^{13} \mathrm{C}$ NMR (100 MHz, $\left.\mathrm{CDCl}_{3}\right): \delta=53.1,126.1,126.5,126.6,127.6,141.5,158.2$; HRMS calculated for $\mathrm{C}_{7} \mathrm{H}_{8} \mathrm{~N}_{2} \mathrm{O}_{2}$ $[\mathrm{M}+\mathrm{H}]^{+}$153.0664, found 153.0662.

6'-(imino(methoxy)methyl)-[2,2'-bipyridine] 1-oxide, Yield: $0.231 \mathrm{~g}(86 \%)$; light yellow powder; mp 120-122 ${ }^{\circ} \mathrm{C} .{ }^{1} \mathrm{H}$ NMR $\left(400 \mathrm{MHz}, \mathrm{CDCl}_{3}\right): \delta=4.02\left(\mathrm{~s}, 3 \mathrm{H}, \mathrm{O}-\mathrm{CH}_{3}\right), 7.30(\mathrm{t}, \mathrm{J}=4 \mathrm{~Hz}$, $1 \mathrm{H}, \mathrm{Ar}), 7.40(\mathrm{t}, \mathrm{J}=8 \mathrm{~Hz}, 1 \mathrm{H}, \mathrm{Ar}), 7.85-7.94(\mathrm{~m}, 2 \mathrm{H}, \mathrm{Ar}), 8.24(\mathrm{dd}, \mathrm{J}=8,4 \mathrm{~Hz}, 1 \mathrm{H}, \mathrm{Ar})$, $8.31(\mathrm{~d}, \mathrm{~J}=8 \mathrm{~Hz}, 1 \mathrm{H}, \mathrm{Ar}), 9.02(\mathrm{~d}, \mathrm{~J}=8 \mathrm{~Hz}, 1 \mathrm{H}, \mathrm{Ar}), 9.20(\mathrm{br} \mathrm{s}, 1 \mathrm{H}, \mathrm{NH}) ;{ }^{13} \mathrm{C}$ NMR $(100 \mathrm{MHz}$, $\left.\mathrm{CDCl}_{3}\right): \delta=54.1,121.6,125.7,125.9,127.1,128.1,137.8,138.4,140.8,147.1,149.2,166.7$; HRMS calculated for $\mathrm{C}_{12} \mathrm{H}_{11} \mathrm{~N}_{3} \mathrm{O}[\mathrm{M}+\mathrm{H}]^{+} 230.0930$, found 230.0932 .

methyl 6-(tert-butyl)picolinimidate (14), Yield: $0.210 \mathrm{~g}(94 \%)$; orange oil; solidifies when stored in the fridge. ${ }^{1} \mathrm{H}$ NMR $\left(400 \mathrm{MHz}, \mathrm{CDCl}_{3}\right): \delta=1.37\left(\mathrm{~s}, 9 \mathrm{H}, \mathrm{C}\left(\mathrm{CH}_{3}\right)_{3}\right), 4.00\left(\mathrm{~s}, 3 \mathrm{H}, \mathrm{CH}_{3}\right)$, $7.41(\mathrm{~d}, \mathrm{~J}=8 \mathrm{~Hz}, 1 \mathrm{H}, \mathrm{Ar}), 7.62(\mathrm{~d}, 1 \mathrm{H}, \mathrm{Ar}), 7.69(\mathrm{t}, \mathrm{J}=8 \mathrm{~Hz}, 1 \mathrm{H}, \mathrm{Ar}) ;{ }^{13} \mathrm{C} \mathrm{NMR}(100 \mathrm{MHz}$, $\left.\mathrm{CDCl}_{3}\right): \delta=30.2,37.7,54.0,117.9,121.1,137.5,145.8,167.6,168.9$; HRMS calculated for $\mathrm{C}_{11} \mathrm{H}_{16} \mathrm{~N}_{2} \mathrm{O}[\mathrm{M}+\mathrm{H}]^{+}$193.1341, found 193.1338.

\subsection{General Procedure for the Preparation of NH-Imidazolines}

Based on the literature procedure [20], after total conversion of the carbonitrile into the imidate conducted as described in the previous section, $(1 R, 2 R)$-cyclohexane-1,2-diamine (100 mg, $0.876 \mathrm{mmol}), \mathrm{Et}_{3} \mathrm{~N}$ (0.32 mL, $\left.4.36 \mathrm{mmol}\right), \mathrm{AcOH}$ ( 3 drops) were added and the resulting solution was stirred at $40^{\circ} \mathrm{C}$ for respective time (given below). The solvent was evaporated in vacuo to obtain a crude product.

2-((3aR,7aR)-3a,4,5,6,7,7a-hexahydro-1H-benzo[d]imidazol-2-yl)pyridine 1-oxide (22), Reaction time: $64 \mathrm{~h}$; purification by column chromatography on silica gel with eluent AcOEt:MeOH:NH $\mathrm{Naq}_{3 \mathrm{y}}, 1: 1: 0.01$; yield: $0.120 \mathrm{~g}(63 \%)$; white solid; $\mathrm{mp} 143{ }^{\circ} \mathrm{C},[\alpha]_{\mathrm{D}}^{20}-30.7$ (c 1.0, MeOH). ${ }^{1} \mathrm{H}$ NMR $\left(400 \mathrm{MHz}, \mathrm{CDCl}_{3}\right): \delta=1.30-1.35\left(\mathrm{~m}, 2 \mathrm{H}, \mathrm{CH}_{2}\right), 1.51-1.53(\mathrm{~m}, 2 \mathrm{H}$, $\left.\mathrm{CH}_{2}\right), 1.81-1.83\left(\mathrm{~m}, 2 \mathrm{H}, \mathrm{CH}_{2}\right), 2.26-2.29\left(\mathrm{~m}, 2 \mathrm{H}, \mathrm{CH}_{2}\right), 3.10-3.12(\mathrm{~m}, 2 \mathrm{H}, 2 \times \mathrm{CH}), 7.29-7.33$ $(\mathrm{m}, 2 \mathrm{H}, \mathrm{Ar}), 8.19(\mathrm{~d}, \mathrm{~J}=8 \mathrm{~Hz}, 1 \mathrm{H}, \mathrm{Ar}), 8.24(\mathrm{dd}, \mathrm{J}=4,4 \mathrm{~Hz}, 1 \mathrm{H}, \mathrm{Ar}) ;{ }^{13} \mathrm{C} \mathrm{NMR}(100 \mathrm{MHz}$, $\left.\mathrm{CDCl}_{3}\right): \delta=25.0$ (br), 30.7, 50.6, 126.4, 126.8, 127.9, 140.4, 140.6, 161.1; HRMS calculated for $\mathrm{C}_{12} \mathrm{H}_{15} \mathrm{~N}_{3} \mathrm{O}[\mathrm{M}+\mathrm{H}]^{+}$218.1293, found 218.1286.

$6^{\prime}-((3 a R, 7 a R)-3 a, 4,5,6,7,7 a-h e x a h y d r o-1 H-b e n z o[d] i m i d a z o l-2-y l)-\left[2,2^{\prime}\right.$-bipyridine] 1-oxide (26), Reaction time: $96 \mathrm{~h}$; after evaporation of methanol, the residue was dissolved in $\mathrm{CHCl}_{3}$ $(10 \mathrm{~mL})$, washed with $\mathrm{NaHCO}_{3}(5 \mathrm{~mL})$, brine and dried over $\mathrm{Na}_{2} \mathrm{SO}_{4} \cdot$; Yield: $21 \mathrm{mg}$ (15\%); brown, gummy liquid; $[\alpha]_{\mathrm{D}}^{20}+54.2(\mathrm{c} 0.18, \mathrm{MeOH}) .{ }^{1} \mathrm{H}$ NMR $\left(400 \mathrm{MHz}, \mathrm{CDCl}_{3}\right)$ : $\delta=1.33-1.38\left(\mathrm{~m}, 2 \mathrm{H}, \mathrm{CH}_{2}\right), 1.56-1.58\left(\mathrm{~m}, 2 \mathrm{H}, \mathrm{CH}_{2}\right), 1.82-1.85\left(\mathrm{~m}, 2 \mathrm{H}, \mathrm{CH}_{2}\right), 2.29-2.32(\mathrm{~m}$, $\left.2 \mathrm{H}, \mathrm{CH}_{2}\right), 3.18-3.20(\mathrm{~m}, 2 \mathrm{H}, 2 \times \mathrm{CH}), 7.27(\mathrm{td}, \mathrm{J}=8,4 \mathrm{~Hz}, 1 \mathrm{H}, \mathrm{Ar}), 7.35(\mathrm{td}, \mathrm{J}=8,4 \mathrm{~Hz}$, $1 \mathrm{H}, \mathrm{Ar}), 7.88(\mathrm{t}, \mathrm{J}=8 \mathrm{~Hz}, 1 \mathrm{H}, \mathrm{Ar}), 8.15(\mathrm{dd}, \mathrm{J}=8,4 \mathrm{~Hz}, 1 \mathrm{H}, \mathrm{Ar}), 8.19(\mathrm{~d}, \mathrm{~J}=8 \mathrm{~Hz}, 1 \mathrm{H}, \mathrm{Ar})$, $8.30(\mathrm{dd}, \mathrm{J}=8,4 \mathrm{~Hz}, 1 \mathrm{H}, \mathrm{Ar}), 8.93(\mathrm{~d}, \mathrm{~J}=8 \mathrm{~Hz}, 1 \mathrm{H}, \mathrm{Ar}) ;{ }^{13} \mathrm{C}$ NMR $\left(100 \mathrm{MHz}, \mathrm{CDCl}_{3}\right)$ : 
$\delta=25.0$ (br), 30.9, 55.6, 122.7, 125.5, 125.7, 127.0, 127.4, 127.9, 137.2, 140.9, 146.9, 148.6, 165.0; HRMS calculated for $\mathrm{C}_{17} \mathrm{H}_{18} \mathrm{~N}_{4} \mathrm{O}[\mathrm{M}+\mathrm{H}]^{+} 295.1559$, found 295.1552 .

\subsection{General Procedure for Cyanation with TMSCN/(CH3 $)_{2} \mathrm{NCOCl}$ System}

According to the described procedure [44]: to a solution of a substrate $(5.7 \mathrm{mmol}, 1 \mathrm{eq}$.) and TMSCN $(0.89 \mathrm{~mL}, 6.25 \mathrm{mmol}, 1.1$ eq. $)$ in dichloromethane $(11.5 \mathrm{~mL}), \mathrm{N}^{\prime} \mathrm{N}^{\prime}$-dimethylcarbamoyl chloride $(0.57 \mathrm{~mL}, 6.25 \mathrm{mmol}, 1.1$ eq.) was added dropwise, The resulting mixture was stirred at room temperature for $6 \mathrm{~d}$. The reaction mixture was then washed with $\mathrm{NaHCO}_{3}(5 \mathrm{~mL})$, brine, and dried over $\mathrm{Na}_{2} \mathrm{SO}_{4}$. The solvent was evaporated to give a crude product.

3,5-dimethylpyridinecarbonitrile (8), Yield: $0.644 \mathrm{~g}(86 \%)$; pale yellow needles; $\mathrm{mp} 58-61{ }^{\circ} \mathrm{C}$ (lit. [45] $\left.61.5^{\circ} \mathrm{C}\right) ;{ }^{1} \mathrm{H}$ NMR $\left(400 \mathrm{MHz}, \mathrm{CDCl}_{3}\right): \delta=2.38\left(\mathrm{~s}, 3 \mathrm{H}, \mathrm{CH}_{3}\right), 2.50\left(\mathrm{~s}, 3 \mathrm{H}, \mathrm{CH}_{3}\right)$, 7.45 (s, $1 \mathrm{H}, \mathrm{Py}), 8.35$ (s, $1 \mathrm{H}, \mathrm{Py})$; spectroscopic data in agreement with literature values [45].

6-tert-butylpyridine-2-carbonitrile (13), Purification by column chromatography on silica gel with eluent $\mathrm{CH}_{2} \mathrm{Cl}_{2}: \mathrm{MeOH}, 10: 1$; yield: $0.935 \mathrm{~g}(89 \%)$; yellow oil; ${ }^{1} \mathrm{H} \mathrm{NMR}(400 \mathrm{MHz}$, $\left.\mathrm{CDCl}_{3}\right): \delta=1.35\left(\mathrm{~s}, 9 \mathrm{H}, \mathrm{C}\left(\mathrm{CH}_{3}\right)_{3}\right), 7.48(\mathrm{dd}, \mathrm{J}=8,1 \mathrm{~Hz}, 1 \mathrm{H}, \mathrm{Py}), 7.54(\mathrm{dd}, \mathrm{J}=8,1 \mathrm{~Hz}, 1 \mathrm{H}$, Py), $7.72(\mathrm{t}, \mathrm{J}=8 \mathrm{~Hz}, 1 \mathrm{H}, \mathrm{Py})$; spectroscopic data in agreement with literature values [38].

\subsection{General Procedure for Preparation of the Substituted Pyridine N-Oxide Oxazolines from Imidates}

Following the procedure used in the preparation of corresponding pyridine oxazolines derivatives [32]: a vial was charged with imidate ( $0.657 \mathrm{mmol}, 1$ eq.), amino alcohol ( $0.657 \mathrm{mmol}, 1$ eq.), and anhydrous toluene $(1.5 \mathrm{~mL})$. The molecular sieves $4 \AA(0.3 \mathrm{~g})$ were added and the vial was sealed. The reaction was then stirred at $70^{\circ} \mathrm{C}$ for 7 days. After this time, the reaction mixture was filtered over celite and concentrated in vacuo to give the pure product.

(S)-2-(4-isopropyl-4,5-dihydrooxazol-2-yl)pyridine 1-oxide (4a), Yield: $90 \mathrm{mg}$ (73\%); off-yellow oil; $[\alpha]_{\mathrm{D}}^{20}-58.0$ (c 1.0, MeOH). ${ }^{1} \mathrm{H}$ NMR $\left(400 \mathrm{MHz}, \mathrm{CDCl}_{3}\right): \delta=0.94(\mathrm{~d}, \mathrm{~J}=8 \mathrm{~Hz}, 3 \mathrm{H}$, $\left.\mathrm{CH}_{3}\right), 1.02\left(\mathrm{~d}, \mathrm{~J}=8 \mathrm{~Hz}, 3 \mathrm{H}, \mathrm{CH}_{3}\right), 1.86-1.94\left(\mathrm{~m}, 1 \mathrm{H}, \mathrm{CH}\left(\mathrm{CH}_{3}\right)_{2}\right), 4.12-4.24\left(\mathrm{~m}, 2 \mathrm{H}, \mathrm{O}-\mathrm{CH}_{2}\right)$, 4.49-4.53 (m, 1H, N-CH), $7.22(\mathrm{t}, \mathrm{J}=4 \mathrm{~Hz}, 1 \mathrm{H}, \mathrm{Ar}), 7.28(\mathrm{td}, \mathrm{J}=4,16 \mathrm{~Hz}, 1 \mathrm{H}, \mathrm{Ar}), 7.67(\mathrm{~d}$, $\mathrm{J}=8 \mathrm{~Hz}, 1 \mathrm{H}, \mathrm{Ar}), 8.23(\mathrm{~d}, \mathrm{~J}=8 \mathrm{~Hz}, 1 \mathrm{H}, \mathrm{Ar}) ;{ }^{13} \mathrm{C} \mathrm{NMR}\left(100 \mathrm{MHz}, \mathrm{CDCl}_{3}\right): \delta=18.2,18.9$, 32.6, 71.1, 72.7, 124.6, 127.2, 128.4, 129.0, 140.9, 158.4. HRMS calculated for $\mathrm{C}_{11} \mathrm{H}_{14} \mathrm{~N}_{2} \mathrm{O}_{2}$ $[\mathrm{M}+\mathrm{H}]^{+}$207.1134, found 207.1129.

(S)-2-(4-(tert-butyl)-4,5-dihydrooxazol-2-yl)pyridine 1-oxide (4b), Yield: $140 \mathrm{mg}(96 \%)$; crystallizing off-yellow oil; mp $83-87^{\circ} \mathrm{C}$; $[\alpha]_{\mathrm{D}}^{20}-79.1$ (c 1.0, $\left.\mathrm{MeOH}\right) .{ }^{1} \mathrm{H} \mathrm{NMR}\left(400 \mathrm{MHz}, \mathrm{CDCl}_{3}\right)$ : $\delta=0.96\left(\mathrm{~s}, 9 \mathrm{H}, 3 \times \mathrm{CH}_{3}\right), 4.09\left(\mathrm{dd}, \mathrm{J}=12,8 \mathrm{~Hz}, 1 \mathrm{H}, \mathrm{O}-\mathrm{CH}_{2}\right), 4.31(\mathrm{t}, \mathrm{J}=8 \mathrm{~Hz}, 1 \mathrm{H}, \mathrm{N}-\mathrm{CH})$, $4.46\left(\mathrm{dd}, \mathrm{J}=12,8 \mathrm{~Hz}, 1 \mathrm{H}, \mathrm{O}-\mathrm{CH}_{2}\right), 7.20-7.30(\mathrm{~m}, 2 \times 1 \mathrm{H}, \mathrm{Py}), 7.65(\mathrm{dd}, \mathrm{J}=8,4 \mathrm{~Hz}, 1 \mathrm{H}$, Ar), $8.21(\mathrm{dd}, \mathrm{J}=8,4 \mathrm{~Hz}, 1 \mathrm{H}, \mathrm{Ar}) ;{ }^{13} \mathrm{C}$ NMR $\left(100 \mathrm{MHz}, \mathrm{CDCl}_{3}\right): \delta=26.0,34.0,69.8,76.1$, 124.6, 127.1, 128.4, 129.1, 140.8, 158.6; HRMS calculated for $\mathrm{C}_{12} \mathrm{H}_{16} \mathrm{~N}_{2} \mathrm{O}_{2}[\mathrm{M}+\mathrm{H}]^{+} 221.1290$, found 221.1286.

(S)-2-(4-phenyl-4,5-dihydrooxazol-2-yl)pyridine 1-oxide (4c), Yield: $237 \mathrm{mg}$ (100\%); yelloworange gummy liquid; $[\alpha]_{\mathrm{D}}^{20}-73.8(\mathrm{c} 1.0, \mathrm{MeOH}) .{ }^{1} \mathrm{H}$ NMR $\left(400 \mathrm{MHz}, \mathrm{CDCl}_{3}\right): \delta=4.40$ $(\mathrm{t}, \mathrm{J}=8 \mathrm{~Hz}, 1 \mathrm{H}, \mathrm{CH}), 4.89\left(\mathrm{dd}, \mathrm{J}=8,12 \mathrm{~Hz}, 1 \mathrm{H}, \mathrm{CH}_{2}\right), 5.44\left(\mathrm{dd}, \mathrm{J}=8,12 \mathrm{~Hz}, 1 \mathrm{H}, \mathrm{CH}_{2}\right)$, 7.24-7.42 (m, 7H, Ar), $7.78(\mathrm{dd}, \mathrm{J}=4,8 \mathrm{~Hz}, 1 \mathrm{H}, \mathrm{Ar}), 8.28(\mathrm{~d}, \mathrm{~J}=4 \mathrm{~Hz}, 1 \mathrm{H}, \mathrm{Ar}) ;{ }^{13} \mathrm{C} \mathrm{NMR}$ $\left(100 \mathrm{MHz}, \mathrm{CDCl}_{3}\right): \delta=56.8,66.7,126.9,127.6,127.9,128.6,129.0,129.1,138.7,140.6,141.4$, 160.0; HRMS calculated for $\mathrm{C}_{14} \mathrm{H}_{12} \mathrm{~N}_{2} \mathrm{O}_{2}[\mathrm{M}+\mathrm{H}]^{+}$241.0977, found 241.0970.

2-((3aR,8aS)-3a,8a-dihydro-8H-indeno[1,2-d]oxazol-2-yl)pyridine 1-oxide (4d), Yield: $159 \mathrm{mg}$ (96\%); crystallizing puce gummy liquid; mp $159-161{ }^{\circ} \mathrm{C} ;[\alpha]_{\mathrm{D}}^{20}+154.1$ (c 1.0, $\left.\mathrm{MeOH}\right)$. ${ }^{1} \mathrm{H}$ NMR $\left(400 \mathrm{MHz}, \mathrm{CDCl}_{3}\right): \delta=3.38-3.54\left(\mathrm{~m}, 2 \mathrm{H}, \mathrm{CH}_{2}\right), 5.60\left(\mathrm{td}, \mathrm{J}=8,4 \mathrm{~Hz}, 1 \mathrm{H}, \mathrm{CH}-\mathrm{CH}_{2}\right)$, $5.81(\mathrm{~d}, \mathrm{~J}=8 \mathrm{~Hz}, 1 \mathrm{H}, \mathrm{CH}), 7.17(\mathrm{t}, \mathrm{J}=8 \mathrm{~Hz}, 1 \mathrm{H}, \mathrm{Py}), 7.22-7.28(\mathrm{~m}, 5 \mathrm{H}, \mathrm{Ar}), 7.54-7.55(\mathrm{~m}$, $1 \mathrm{H}, \mathrm{Py}) 7.63(\mathrm{~d}, \mathrm{~J}=8 \mathrm{~Hz}, 1 \mathrm{H}, \mathrm{Ar}), 8.21(\mathrm{~d}, \mathrm{~J}=4 \mathrm{~Hz}, 1 \mathrm{H}, \mathrm{Ar}) ;{ }^{13} \mathrm{C}$ NMR $\left(100 \mathrm{MHz}, \mathrm{CDCl}_{3}\right)$ : $\delta=21.6,39.8,84.1,124.5,125.5,125.8,127.2,127.6,128.3,128.7,128.8,129.1,139.9,141.0$, 158.8. HRMS calculated for $\mathrm{C}_{15} \mathrm{H}_{12} \mathrm{~N}_{2} \mathrm{O}_{2}[\mathrm{M}+\mathrm{H}]^{+} 253.0977$, found 253.0978. 
2-((3aR,4S,7R,7aS)-7,8,8-trimethyl-3a,4,5,6,7,7a-hexahydro-4,7-methanobenzo[d]oxazol-2-yl)pyridine 1-oxide (4e), Recrystallized from ethyl acetate; yield: $100 \mathrm{mg}$ (37\%); white solid; $\mathrm{mp} 136-139{ }^{\circ} \mathrm{C} ;[\alpha]_{\mathrm{D}}^{20}-10.1(\mathrm{c} 1.0, \mathrm{MeOH}) .{ }^{1} \mathrm{H} \mathrm{NMR}\left(400 \mathrm{MHz}, \mathrm{CDCl}_{3}\right): \delta=0.86(\mathrm{~s}, 3 \mathrm{H}$, $\left.\mathrm{CH}_{3}\right), 0.98\left(\mathrm{~s}, 3 \mathrm{H}, \mathrm{CH}_{3}\right), 1.05-1.11\left(\mathrm{~m}, 2 \mathrm{H}, \mathrm{CH}_{2}\right), 1.06\left(\mathrm{~s}, 3 \mathrm{H}, \mathrm{CH}_{3}\right), 1.49-1.57(\mathrm{~m}, 1 \mathrm{H}, \mathrm{CH})$, 1.73-1.80 (m, 1H, CH), $2.22(\mathrm{~d}, \mathrm{~J}=4 \mathrm{~Hz}, 1 \mathrm{H}, \mathrm{CH}), 4.28(\mathrm{~d}, \mathrm{~J}=12 \mathrm{~Hz}, 1 \mathrm{H}, \mathrm{CH}), 4.52(\mathrm{~d}$, $\mathrm{J}=8 \mathrm{~Hz}, 1 \mathrm{H}, \mathrm{CH}), 7.21(\mathrm{t}, \mathrm{J}=8 \mathrm{~Hz}, 1 \mathrm{H}, \mathrm{Ar}), 7.28(\mathrm{t}, \mathrm{J}=6 \mathrm{~Hz}, 1 \mathrm{H}, \mathrm{Ar}), 7.62(\mathrm{dd}, \mathrm{J}=4,8 \mathrm{~Hz}$, $1 \mathrm{H}, \mathrm{Ar}), 8.23(\mathrm{~d}, \mathrm{~J}=8 \mathrm{~Hz}, 1 \mathrm{H}, \mathrm{Ar}) ;{ }^{13} \mathrm{C} \mathrm{NMR}\left(100 \mathrm{MHz}, \mathrm{CDCl}_{3}\right): \delta=11.4,19.1,23.5,26.2$, $32.1,47.2$, 48.9, 49.0, 92.0, 124.5, 127.0, 128.2, 141.2, 159.4; HRMS calculated for $\mathrm{C}_{16} \mathrm{H}_{20} \mathrm{~N}_{2} \mathrm{O}_{2}$ $[\mathrm{M}+\mathrm{H}]^{+}$273.1603, found 273.1602.

(S)-6'-(4-isopropyl-4,5-dihydrooxazol-2-yl)-[2,2'-bipyridine] 1-oxide (5a), Yield after $24 \mathrm{h:} 45 \mathrm{mg}$ (22\%); crystallizing yellowish liquid; mp $121-125^{\circ} \mathrm{C} ;[\alpha]_{\mathrm{D}}^{20}-11.0$ (c 1.0, MeOH). ${ }^{1} \mathrm{H}$ NMR $\left(400 \mathrm{MHz}, \mathrm{CDCl}_{3}\right): \delta=0.94\left(\mathrm{~d}, \mathrm{~J}=8 \mathrm{~Hz}, 3 \mathrm{H}, \mathrm{CH}_{3}\right), 1.05\left(\mathrm{~d}, \mathrm{~J}=8 \mathrm{~Hz}, 3 \mathrm{H}, \mathrm{CH}_{3}\right), 1.86-1.95(\mathrm{~m}$, $\left.1 \mathrm{H}, \mathrm{CH}\left(\mathrm{CH}_{3}\right)_{2}\right), 4.14-4.25\left(\mathrm{~m}, 2 \mathrm{H}, \mathrm{CH}_{2}\right), 4.52(\mathrm{dd}, \mathrm{J}=8,12 \mathrm{~Hz}, 1 \mathrm{H}, \mathrm{N}-\mathrm{CH}), 7.24-7.28(\mathrm{~m}, 1 \mathrm{H}$, Ar), 7.36 (t, J = $8 \mathrm{~Hz}, 1 \mathrm{H}, \mathrm{Ar}), 7.90$ (t, J = 8 Hz, 1H, Ar), 8.12 (d, J = 8 Hz, 1H, Ar), 8.24 (d, $\mathrm{J}=8 \mathrm{~Hz}, 1 \mathrm{H}, \mathrm{Ar}), 8.29(\mathrm{~d}, \mathrm{~J}=4 \mathrm{~Hz}, 1 \mathrm{H}, \mathrm{Ar}), 8.96(\mathrm{~d}, \mathrm{~J}=8 \mathrm{~Hz}, 1 \mathrm{H}, \mathrm{Ar}) ;{ }^{13} \mathrm{C} \mathrm{NMR}(100 \mathrm{MHz}$, $\left.\mathrm{CDCl}_{3}\right): \delta=18.2,19.2,32.9,70.9,73.0,124.7,125.6,126.0,127.3,128.5,137.0,140.6,146.8$, $146.9,149.7,162.4$. HRMS calculated for $\mathrm{C}_{16} \mathrm{H}_{17} \mathrm{~N}_{3} \mathrm{O}_{2}[\mathrm{M}+\mathrm{H}]^{+} 284.1399$, found 284.1421.

(S)-6'-(4-(tert-butyl)-4,5-dihydrooxazol-2-yl)-[2,2'-bipyridine] 1-oxide (5b), Yield: $135 \mathrm{mg}(96 \%)$; yellow gummy liquid; $[\alpha]_{\mathrm{D}}^{20}-7.8$ (c 1.0, $\left.\mathrm{MeOH}\right) .{ }^{1} \mathrm{H} \mathrm{NMR}\left(400 \mathrm{MHz}, \mathrm{CDCl}_{3}\right): \delta=0.97$ (s, $\left.9 \mathrm{H}, 3 \times \mathrm{CH}_{3}\right), 4.12\left(\mathrm{dd}, \mathrm{J}=12,8 \mathrm{~Hz}, 1 \mathrm{H}, \mathrm{O}-\mathrm{CH}_{2}\right), 4.32(\mathrm{t}, \mathrm{J}=8 \mathrm{~Hz}, 1 \mathrm{H}, \mathrm{N}-\mathrm{CH}), 4.46$ (dd, $\left.\mathrm{J}=12,8 \mathrm{~Hz}, 1 \mathrm{H}, \mathrm{O}-\mathrm{CH}_{2}\right), 7.22-7.28(\mathrm{~m}, 2 \times 1 \mathrm{H}, \mathrm{Py}), 7.34-7.38(\mathrm{t}, \mathrm{J}=8 \mathrm{~Hz}, 1 \mathrm{H}, \mathrm{Ar}), 7.90(\mathrm{t}$, $\mathrm{J}=8 \mathrm{~Hz}, 1 \mathrm{H}, \mathrm{Ar}), 8.16(\mathrm{~d}, \mathrm{~J}=8 \mathrm{~Hz}, 1 \mathrm{H}, \mathrm{Ar}), 8.23(\mathrm{dd}, \mathrm{J}=8,4 \mathrm{~Hz}, 1 \mathrm{H}, \mathrm{Ar}), 8.29$ (d, J = $8 \mathrm{~Hz}$, $1 \mathrm{H}, \mathrm{Ar}) ;{ }^{13} \mathrm{C} \mathrm{NMR}\left(100 \mathrm{MHz} \mathrm{CDCl}_{3}\right): \delta=26.0,34.1,69.5,76.5,124.8,125.4,125.6,126.0$, $127.3,128.3,128.5,129.1,137.0,146.6,162.4$; HRMS calculated for $\mathrm{C}_{17} \mathrm{H}_{19} \mathrm{~N}_{3} \mathrm{O}_{2}[\mathrm{M}+\mathrm{H}]^{+}$ 298.1555, found 298.1551.

(S)-6'-(4-phenyl-4,5-dihydrooxazol-2-yl)-[2,2'-bipyridine] 1-oxide (5c), Yield: $90 \mathrm{mg}(100 \%)$; yellow gummy liquid; $[\alpha]_{\mathrm{D}}^{20}-74.6\left(\mathrm{c} 1.0, \mathrm{CHCl}_{3}\right) .{ }^{1} \mathrm{H} \mathrm{NMR}\left(400 \mathrm{MHz}, \mathrm{CDCl}_{3}\right): \delta=4.39(\mathrm{t}$, $\mathrm{J}=8 \mathrm{~Hz}, 1 \mathrm{H}, \mathrm{CH}), 4.90\left(\mathrm{t}, \mathrm{J}=8 \mathrm{~Hz}, 1 \mathrm{H}, \mathrm{CH}_{2}\right), 5.46\left(\mathrm{t}, \mathrm{J}=8,1 \mathrm{H}, \mathrm{CH}_{2}\right), 7.22-7.40(\mathrm{~m}, 7 \mathrm{H}, \mathrm{Ar})$, $7.94(\mathrm{t}, \mathrm{J}=8 \mathrm{~Hz}, 1 \mathrm{H}, \mathrm{Ar}), 8.19-8.31(\mathrm{~m}, 3 \mathrm{H}, \mathrm{Ar}), 8.99$ (d, J = 8 Hz, 1H, Ar); ${ }^{13} \mathrm{C} \mathrm{NMR}(100 \mathrm{MHz}$, $\left.\mathrm{CDCl}_{3}\right): \delta=70.5,75.6,125.0,125.4,125.7,126.1,126.9,127.6,127.9,128.3,128.5,128.7,128.9$, 129.1, 137.1, 140.6, 141.8, 146.5, 163.8; HRMS calculated for $\mathrm{C}_{19} \mathrm{H}_{15} \mathrm{~N}_{3} \mathrm{O}_{2}[\mathrm{M}+\mathrm{H}]^{+} 318.1242$, found 318.1247 .

$6^{\prime}-((3 a R, 8 a S)-3 a, 8 a-d i h y d r o-8 H$-indeno[1,2-d]oxazol-2-yl)-[2,2'-bipyridine] 1-oxide (5d), Yield: $81 \mathrm{mg}(96 \%)$; puce solid; mp $180-184{ }^{\circ} \mathrm{C} ;[\alpha]_{\mathrm{D}}^{20}+43.5$ (c 1.0, MeOH). ${ }^{1} \mathrm{H} \mathrm{NMR}(400 \mathrm{MHz}$, $\left.\mathrm{CDCl}_{3}\right): \delta=3.43-3.56\left(\mathrm{~m}, 2 \mathrm{H}, \mathrm{CH}_{2}\right), 5.59\left(\mathrm{td}, \mathrm{J}=8,4 \mathrm{~Hz}, 1 \mathrm{H}, \mathrm{CH}-\mathrm{CH}_{2}\right), 5.82(\mathrm{~d}, \mathrm{~J}=8 \mathrm{~Hz}$, 1H, CH), 7.22-7.30 (m, 5H, Ar), 7.35 (t, J = 8 Hz, 1H, Py), 7.57-7.60 (m, 1H, Py) 7.86 (d, $\mathrm{J}=4 \mathrm{~Hz}, 1 \mathrm{H}, \mathrm{Py}), 8.06$ (d, J = $8 \mathrm{~Hz}, 1 \mathrm{H}, \mathrm{Py}), 8.21(\mathrm{~d}, \mathrm{~J}=8 \mathrm{~Hz}, 1 \mathrm{H}, \mathrm{Py}), 8.27$ (d, J = $8 \mathrm{~Hz}, 1 \mathrm{H}$, Py), 8.91 (d, J = 8 Hz, 1H, Py); ${ }^{13} \mathrm{C}$ NMR (100 MHz, $\left.\mathrm{CDCl}_{3}\right): \delta=39.9,50.8,84.2,124.8,125.4$, 125.7, 125.8, 126.2, 127.4, 127.6, 128.6, 128.8, 137.0, 139.8, 140.6, 141.6, 146.7, 146.9, 149.8, 163.1; HRMS calculated for $\mathrm{C}_{20} \mathrm{H}_{15} \mathrm{~N}_{3} \mathrm{O}_{2}[\mathrm{M}+\mathrm{H}]^{+} 330.1242$, found 330.1244 .

(S)-2-(6-(tert-butyl)pyridin-2-yl)-4-isopropyl-4,5-dihydrooxazole (15a), Yield: $209 \mathrm{mg}$ (100\%); yellow oil; ${ }^{1} \mathrm{H} \mathrm{NMR}\left(\mathrm{CDCl}_{3}\right): \delta=0.93\left(\mathrm{~d}, \mathrm{~J}=8 \mathrm{~Hz}, 3 \mathrm{H}, \mathrm{CH}_{3}\right), 1.04(\mathrm{~d}, \mathrm{~J}=8 \mathrm{~Hz}, 3 \mathrm{H}$, $\left.\mathrm{CH}_{3}\right), 1.38\left(\mathrm{~s}, 9 \mathrm{H}, \mathrm{C}\left(\mathrm{CH}_{3}\right)_{3}\right), 1.85-1.90\left(\mathrm{~m}, 1 \mathrm{H}, \mathrm{CH}\left(\mathrm{CH}_{3}\right)_{2}\right), 4.10-4.15(\mathrm{~m}, 1 \mathrm{H} \mathrm{N}-\mathrm{CH}), 4.21(\mathrm{t}$, $\left.\mathrm{J}=8 \mathrm{~Hz}, 1 \mathrm{H}, \mathrm{O}-\mathrm{CH}_{2}\right), 4.50\left(\mathrm{dd}, \mathrm{J}=4,4 \mathrm{~Hz}, 1 \mathrm{H}, \mathrm{O}-\mathrm{CH}_{2}\right), 7.42(\mathrm{dd}, \mathrm{J}=8,4 \mathrm{~Hz}, 1 \mathrm{H}, \mathrm{Py})$, $7.65(\mathrm{t}, \mathrm{J}=8 \mathrm{~Hz}, 1 \mathrm{H}, \mathrm{Py}), 7.88(\mathrm{~d}, \mathrm{~J}=8 \mathrm{~Hz}, 1 \mathrm{H}, \mathrm{Py})$; spectroscopic data consistent with literature values [22].

(S)-4-(tert-butyl)-2-(6-(tert-butyl)pyridin-2-yl)-4,5-dihydrooxazole (15b), yield: $271 \mathrm{mg}(100 \%)$; yellow oil; ${ }^{1} \mathrm{H}$ NMR $\left(\mathrm{CDCl}_{3}\right): \delta=0.96\left(\mathrm{~s}, 9 \mathrm{H}, \mathrm{C}\left(\mathrm{CH}_{3}\right)_{3}\right), 1.38\left(\mathrm{~s}, 9 \mathrm{H}, \mathrm{Py}-\mathrm{C}\left(\mathrm{CH}_{3}\right)_{3}\right), 4.09$ (dd, $\left.\mathrm{J}=12,8 \mathrm{~Hz}, 1 \mathrm{H}, \mathrm{O}-\mathrm{CH}_{2}\right), 4.30(\mathrm{t}, \mathrm{J}=8 \mathrm{~Hz}, 1 \mathrm{H}, \mathrm{N}-\mathrm{CH}), 4.45\left(\mathrm{dd}, \mathrm{J}=8,8 \mathrm{~Hz}, 1 \mathrm{H}, \mathrm{O}-\mathrm{CH}_{2}\right)$, $7.41(\mathrm{~d}, \mathrm{~J}=8 \mathrm{~Hz}, 1 \mathrm{H}, \mathrm{Py}), 7.65$ (t, J = 8 Hz, 1H, Py), 7.91 (dd, J = 8, 4 Hz 1H, Py); spectroscopic data consistent with literature values values [35]. 
(S)-2-(6-(tert-butyl)pyridin-2-yl)-4-phenyl-4,5-dihydrooxazole (15c), Yield: $254 \mathrm{mg}(88 \%)$; orange gummy liquid; $[\alpha]_{\mathrm{D}}^{20}-21.6$ (c 1.0, $\left.\mathrm{MeOH}\right) .{ }^{1} \mathrm{H} \mathrm{NMR}\left(400 \mathrm{MHz}, \mathrm{CDCl}_{3}\right): \delta=1.41(\mathrm{~s}, 9 \mathrm{H}$, $\left.\mathrm{C}\left(\mathrm{CH}_{3}\right)_{3}\right), 4.36(\mathrm{t}, \mathrm{J}=8,1 \mathrm{H}, \mathrm{N}-\mathrm{CH}), 4.89\left(\mathrm{dd}, \mathrm{J}=4,4 \mathrm{~Hz}, 1 \mathrm{H}, \mathrm{O}-\mathrm{CH}_{2}\right), 4.89(\mathrm{dd}, \mathrm{J}=12,8 \mathrm{~Hz}$, $\left.1 \mathrm{H}, \mathrm{O}-\mathrm{CH}_{2}\right), 7.25-7.37(\mathrm{~m}, 5 \mathrm{H}, \mathrm{Ar}), 7.46(\mathrm{~d}, \mathrm{~J}=8 \mathrm{~Hz}, 1 \mathrm{H}, \mathrm{Py}), 7.69(\mathrm{t}, \mathrm{J}=8 \mathrm{~Hz}, 1 \mathrm{H}, \mathrm{Py})$, $7.98(\mathrm{~d}, \mathrm{~J}=8 \mathrm{~Hz}, 1 \mathrm{H}, \mathrm{Py}) ;{ }^{13} \mathrm{C}$ NMR $\left(100 \mathrm{MHz}, \mathrm{CDCl}_{3}\right): \delta=30.3,37.8,70.3,75.5,121.5$, 121.6, 126.5, 127.0, 127.7, 128.7, 128.8, 136.7, 142.2, 145.7, 164.8, 169.6; HRMS calculated for $\mathrm{C}_{18} \mathrm{H}_{20} \mathrm{~N}_{2} \mathrm{O}[\mathrm{M}+\mathrm{H}]^{+}$281.1654, found 281.1656.

(3aR,8aS)-2-(6-(tert-butyl)pyridin-2-yl)-3a,8a-dihydro-8H-indeno[1,2-d]oxazole (15d), Yield: $304 \mathrm{mg}(100 \%)$; orange gummy liquid; $[\alpha]_{\mathrm{D}}^{20}+187.4$ (c 1.0, $\left.\mathrm{CHCl}_{3}\right) .{ }^{1} \mathrm{H} \mathrm{NMR}(400 \mathrm{MHz}$, $\left.\mathrm{CDCl}_{3}\right): \delta=1.37\left(\mathrm{~s}, 9 \mathrm{H}, \mathrm{C}\left(\mathrm{CH}_{3}\right)_{3}\right), 3.41-3.54\left(\mathrm{~m}, 2 \mathrm{H}, \mathrm{CH}_{2}\right), 5.55-5.59(\mathrm{~m}, 1 \mathrm{H}, \mathrm{CH}), 5.79(\mathrm{~d}$, $\mathrm{J}=8 \mathrm{~Hz}, 1 \mathrm{H}, \mathrm{CH}), 7.23-7.27(\mathrm{~m}, 4 \mathrm{H}, \mathrm{Ar}), 7.38(\mathrm{~d}, \mathrm{~J}=8 \mathrm{~Hz}, 1 \mathrm{H}, \mathrm{Py}) 7.58-7.60(\mathrm{~m}, 1 \mathrm{H}, \mathrm{Ar})$, $7.61(\mathrm{t}, \mathrm{J}=8 \mathrm{~Hz}, 1 \mathrm{H}, \mathrm{Py}), 7.81(\mathrm{dd}, \mathrm{J}=8,4 \mathrm{~Hz}, 1 \mathrm{H}, \mathrm{Py}) .{ }^{13} \mathrm{C} \mathrm{NMR}\left(100 \mathrm{MHz}, \mathrm{CDCl}_{3}\right): \delta=30.3$, $37.8,40.0,83.7,121.1,121.5,125.4,125.8,127.5,128.6,129.1,136.5,140.0,142.0,146.0,164.0$, 169.4; HRMS calculated for $\mathrm{C}_{19} \mathrm{H}_{20} \mathrm{~N}_{2} \mathrm{O}[\mathrm{M}+\mathrm{H}]^{+}$293.1654, found 293.1653.

(3aR,4S,7R,7aS)-2-(6-(tert-butyl)pyridin-2-yl)-7,8,8-trimethyl-3a,4,5,6,7,7a-hexahydro-4,7-methanobenzo[d]oxazole (15e), Purified by column chromatography with eluent $\mathrm{CH}_{2} \mathrm{Cl}_{2}: \mathrm{MeOH}$, 10:1; yield: $150 \mathrm{mg}(54 \%)$; crystallizing yellow liquid; $\mathrm{mp} 116-119^{\circ} \mathrm{C}[\alpha]_{\mathrm{D}}^{20}-17.8$ (c 1.0, $\left.\mathrm{CHCl}_{3}\right) .{ }^{1} \mathrm{H}$ NMR $\left(400 \mathrm{MHz}, \mathrm{CDCl}_{3}\right): \delta=0.86\left(\mathrm{~s}, 3 \mathrm{H}, \mathrm{CH}_{3}\right), 0.93\left(\mathrm{~s}, 3 \mathrm{H}, \mathrm{CH}_{3}\right), 1.05-1.12(\mathrm{~m}$, $\left.2 \mathrm{H}, \mathrm{CH}_{2}\right), 1.11\left(\mathrm{~s}, 3 \mathrm{H}, \mathrm{CH}_{3}\right), 1.37\left(\mathrm{~s}, 9 \mathrm{H}, \mathrm{C}\left(\mathrm{CH}_{3}\right)_{3}\right), 1.49-1.57(\mathrm{~m}, 1 \mathrm{H}, \mathrm{CH}), 1.73-1.80(\mathrm{~m}, 1 \mathrm{H}$, $\mathrm{CH}), 2.22(\mathrm{~d}, \mathrm{~J}=4 \mathrm{~Hz}, 1 \mathrm{H}, \mathrm{CH}), 4.23(\mathrm{~d}, \mathrm{~J}=8 \mathrm{~Hz}, 1 \mathrm{H}, \mathrm{CH}), 4.46-4.49(\mathrm{~m}, 1 \mathrm{H}, \mathrm{CH}), 7.40(\mathrm{~d}$, $\mathrm{J}=8 \mathrm{~Hz}, 1 \mathrm{H}, \mathrm{Py}), 7.62-7.66(\mathrm{~m}, 1 \mathrm{H}, \mathrm{Py}), 7.70-7.73(\mathrm{~m}, 1 \mathrm{H}, \mathrm{Py}) ;{ }^{13} \mathrm{C} \mathrm{NMR}\left(100 \mathrm{MHz}, \mathrm{CDCl}_{3}\right)$ : $\delta=11.4,11.9,18.8,23.6,26.2,30.0,30.3,32.2,37.8,47.2,48.8,49.0,91.9,121.1,121.2,128.3$, 136.6, 169.6; HRMS calculated for $\mathrm{C}_{20} \mathrm{H}_{28} \mathrm{~N}_{2} \mathrm{O}[\mathrm{M}+\mathrm{H}]^{+} 313.2280$, found 313.2290.

3.7. General Procedure for Preparation of $\mathbf{1 0 a}-\mathbf{d}, \mathbf{1 9 a}-\mathbf{e}, \mathbf{2 0 c}, \mathbf{e}$ and $\mathbf{2 1 - I s o q u i n o l i n e , ~ I s o q u i n o l i n e ~}$ $\mathrm{N}$-Oxide, and 3,5-Lutidine N-Oxide Oxazolines

According to the procedure described by Cornejo et al. [36]: a round-bottomed flask fitted with a reflux condenser was charged with carbonitrile $(0.973 \mathrm{mmol}, 1$ eq. $)$ and zinc triflate $(15 \% \mathrm{~mol}, 10 \% \mathrm{~mol}$ to obtain $20 \mathrm{c}$ from 18 or $1 \%$ mol to obtain $19 \mathrm{c}$ from 17$)$. The system was purged with argon and dry toluene $(9.8 \mathrm{~mL})$ was added. The solution was stirred for $5 \mathrm{~min}$ and then amino alcohol $(0.973 \mathrm{mmol}, 1$ eq.) was added. The solution was heated under reflux and monitored with TLC. After completion, the reaction mixture was allowed to cool, and diluted with EtOAc. The solution was then washed three times with $\mathrm{NaHCO}_{3}(3 \times 10 \mathrm{~mL})$, and dried with $\mathrm{MgSO}_{4}$. The solvent evaporated to give the pure product $19 \mathrm{~b}$. Crude products $19 \mathrm{a}, \mathrm{c}-\mathrm{e}$ were purified by column chromatography on silica gel.

(S)-4-isopropyl-2-(isoquinolin-1-yl)-4,5-dihydrooxazole (19a), Reaction time: $72 \mathrm{~h}$; TLC and purification via column chromatography on silica gel with eluent $\mathrm{CHCl}_{3}: \mathrm{MeOH}, 12: 1$; yield: $131 \mathrm{mg}(86 \%) ;{ }^{1} \mathrm{H} \mathrm{NMR}\left(400 \mathrm{MHz}, \mathrm{CDCl}_{3}\right): \delta=1.03\left(\mathrm{~d}, \mathrm{~J}=8 \mathrm{~Hz}, 3 \mathrm{H}, \mathrm{CH}_{3}\right), 1.13(\mathrm{~d}, \mathrm{~J}=8 \mathrm{~Hz}$, $\left.3 \mathrm{H}, \mathrm{CH}_{3}\right), 1.93-2.05\left(\mathrm{~m}, 1 \mathrm{H}, \mathrm{CH}\left(\mathrm{CH}_{3}\right)_{2}\right), 4.24\left(\mathrm{t}, \mathrm{J}=8 \mathrm{~Hz}, 1 \mathrm{H}, \mathrm{O}-\mathrm{CH}_{2}\right), 4.31-4.36(\mathrm{~m}, 1 \mathrm{H}$, $\mathrm{N}-\mathrm{CH}), 4.54\left(\mathrm{dd}, \mathrm{J}=8,4 \mathrm{~Hz}, 1 \mathrm{H}, \mathrm{O}-\mathrm{CH}_{2}\right), 7.65-7.73(\mathrm{~m}, 2 \mathrm{H}, \mathrm{Ar}), 7.74(\mathrm{~d}, \mathrm{~J}=4 \mathrm{~Hz}, 1 \mathrm{H}$, Ar), $7.85(\mathrm{~d}, \mathrm{~J}=8 \mathrm{~Hz}, 1 \mathrm{H}, \mathrm{Ar}), 8.62(\mathrm{~d}, \mathrm{~J}=8 \mathrm{~Hz}, 1 \mathrm{H}, \mathrm{Ar}), 9.26(\mathrm{dd}, \mathrm{J}=4,4 \mathrm{~Hz}, 1 \mathrm{H}, \mathrm{Ar})$; spectroscopic data in agreement with literature data values [46].

(S)-4-(tert-butyl)-2-(isoquinolin-1-yl)-4,5-dihydrooxazole (19b), Reaction time: $48 \mathrm{~h}$; yield: $245 \mathrm{mg}(99 \%) ;{ }^{1} \mathrm{H}$ NMR $\left.400 \mathrm{MHz}, \mathrm{CDCl}_{3}\right): \delta=1.05\left(\mathrm{~s}, 9 \mathrm{H}, \mathrm{C}\left(\mathrm{CH}_{3}\right)_{3}\right), 4.27-4.35(\mathrm{~m}, 2 \mathrm{H}$, O- $\left.\mathrm{CH}_{2}\right), 4.46-4.52(\mathrm{~m}, 1 \mathrm{H}, \mathrm{N}-\mathrm{CH}), 7.66-7.73(\mathrm{~m}, 2 \mathrm{H}, \mathrm{Ar}), 7.74(\mathrm{~d}, \mathrm{~J}=4 \mathrm{~Hz}, 1 \mathrm{H}, \mathrm{Ar}), 7.85(\mathrm{~d}$, $\mathrm{J}=8 \mathrm{~Hz}, 1 \mathrm{H}, \mathrm{Ar}), 8.62(\mathrm{~d}, \mathrm{~J}=4 \mathrm{~Hz}, 1 \mathrm{H}, \mathrm{Ar}), 9.30(\mathrm{~d}, \mathrm{~J}=8 \mathrm{~Hz}, 1 \mathrm{H}, \mathrm{Ar})$; spectroscopic data in agreement with literature data [47].

(S)-2-(isoquinolin-1-yl)-4-phenyl-4,5-dihydrooxazole (19c), Reaction time: $72 \mathrm{~h}$; TLC and purification via column chromatography on silica gel with eluent $\mathrm{AcOEt}: \mathrm{CHCl}_{3}: \mathrm{MeOH}$, 10:1:0.1; yield: $27 \mathrm{mg}(10 \%) ;{ }^{1} \mathrm{H}$ NMR $\left(400 \mathrm{MHz}, \mathrm{CDCl}_{3}\right): \delta=4.39(\mathrm{t}, \mathrm{J}=8 \mathrm{~Hz}, 1 \mathrm{H}, \mathrm{N}-\mathrm{CH})$, $4.92\left(\mathrm{dd}, \mathrm{J}=12,8 \mathrm{~Hz}, 1 \mathrm{H}, \mathrm{O}-\mathrm{CH}_{2}\right), 5.64\left(\mathrm{dd}, \mathrm{J}=12,8 \mathrm{~Hz}, 1 \mathrm{H}, \mathrm{O}-\mathrm{CH}_{2}\right), 7.33-7.29(\mathrm{~m}, 1 \mathrm{H}$, 
$\mathrm{Ph})$, 7.37-7.41 (m, 4H, Ph), 7.63-7.73 (m, 2H, Ar), $7.78(\mathrm{~d}, \mathrm{~J}=4 \mathrm{~Hz}, 1 \mathrm{H}, \mathrm{Ar}), 7.85(\mathrm{~d}, \mathrm{~J}=8 \mathrm{~Hz}$, $1 \mathrm{H}, \mathrm{Ar}), 8.67(\mathrm{~d}, \mathrm{~J}=4 \mathrm{~Hz}, 1 \mathrm{H}, \mathrm{Ar}), 9.35(\mathrm{~d}, \mathrm{~J}=8 \mathrm{~Hz}, 1 \mathrm{H}, \mathrm{Ar})$; spectroscopic data in agreement with literature data [46].

(Z)-N,N'-bis((S)-2-hydroxy-1-phenylethyl) isoquinoline-1-carboximidamide (21), Yield: $100 \mathrm{mg}$ (25\%); white solid, mp $105-108{ }^{\circ} \mathrm{C} ;[\alpha]_{\mathrm{D}}^{20}+68.4$ (c 1.0, MeOH). ${ }^{1} \mathrm{H}$ NMR $\left(400 \mathrm{MHz}, \mathrm{CDCl}_{3}\right)$ : $\delta=2.37(\mathrm{br} \mathrm{s}, 2 \mathrm{H}, 2 \times \mathrm{OH}), 3.01(\mathrm{dd}, \mathrm{J}=12,4 \mathrm{~Hz}, 1 \mathrm{H}, \mathrm{CH}), 3.11(\mathrm{dd}, \mathrm{J}=12,8 \mathrm{~Hz}, 1 \mathrm{H}, \mathrm{CH})$, $3.53(\mathrm{dd}, \mathrm{J}=12,12 \mathrm{~Hz}, 1 \mathrm{H}, \mathrm{CH}), 3.72(\mathrm{dd}, \mathrm{J}=12,4 \mathrm{~Hz}, 1 \mathrm{H}, \mathrm{CH}), 3.93(\mathrm{dd}, \mathrm{J}=8,4 \mathrm{~Hz}, 1 \mathrm{H}$, CH) 5.38-5.43 (m, 1H, CH), 7.24-7.28 (m, 5H, Ar), 7.29-7.36 (m, 5H, Ar), 7.63-7.72 (m, 2H, Ar), $7.83(\mathrm{dd}, \mathrm{J}=12,8 \mathrm{~Hz}, 2 \mathrm{H}, \mathrm{Ar}), 8.50(\mathrm{~d}, \mathrm{~J}=4 \mathrm{~Hz}, 1 \mathrm{H}, \mathrm{Ar}), 8.99(\mathrm{~d}, \mathrm{~J}=8 \mathrm{~Hz}, 1 \mathrm{H}, \mathrm{NH})$, $9.54(\mathrm{~d}, \mathrm{~J}=8 \mathrm{~Hz}, 1 \mathrm{H}, \mathrm{Ar}) ;{ }^{13} \mathrm{C}$ NMR $\left(100 \mathrm{MHz}, \mathrm{CDCl}_{3}\right): \delta=14.3,21.2,52.4,52.6,60.5,64.2$, $68.9,124.7,126.5,126.9,127.2,127.2,127.7,127.9,127.9,128.6,128.7,128.8,128.8,128.9$, 130.7, 137.6, 140.3, 140.4, 148.2, 166.2; HRMS calculated for $\mathrm{C}_{26} \mathrm{H}_{25} \mathrm{~N}_{3} \mathrm{O}_{2}[\mathrm{M}+\mathrm{H}]^{+} 412.2025$, found 412.2016 .

(3aR,8aS)- 2-(isoquinolin-1-yl)-3a,8a-dihydro-8H-indeno[1,2-d]oxazole (19d), Reaction time: 72 h; TLC and purification via column chromatography on silica gel with eluent $\mathrm{AcOEt}: \mathrm{CHCl}_{3}$ : $\mathrm{MeOH}, 10: 1: 0.1$; yield: $130 \mathrm{mg}(47 \%) ;{ }^{1} \mathrm{H}$ NMR $\left(400 \mathrm{MHz}, \mathrm{CDCl}_{3}\right): \delta=3.56(\mathrm{~d}, \mathrm{~J}=4 \mathrm{~Hz}, 2 \mathrm{H}$, $\left.\mathrm{CH}_{2}\right), 5.60(\mathrm{~m}, 1 \mathrm{H}, \mathrm{O}-\mathrm{CH}), 5.96(\mathrm{~d}, \mathrm{~J}=8 \mathrm{~Hz}, 1 \mathrm{H}, \mathrm{N}-\mathrm{CH}), 7.26-7.33(\mathrm{~m}, 3 \mathrm{H}, \mathrm{Ar}), 7.60-7.69$ $(\mathrm{m}, 3 \mathrm{H}, \mathrm{Ar}), 7.72(\mathrm{~d}, \mathrm{~J}=4 \mathrm{~Hz}, 1 \mathrm{H}, \mathrm{Ar}), 7.81(\mathrm{~d}, \mathrm{~J}=8 \mathrm{~Hz}, 1 \mathrm{H}, \mathrm{Ar}), 8.61(\mathrm{~d}, \mathrm{~J}=4 \mathrm{~Hz}, 1 \mathrm{H}, \mathrm{Ar})$, $9.18(\mathrm{~d}, \mathrm{~J}=8 \mathrm{~Hz}, 1 \mathrm{H}, \mathrm{Ar})$; spectroscopic data in agreement with literature data [48].

(3aR,4S,7R,7aS)-2-(isoquinolin-1-yl)-7,8,8-trimethyl-3a,4,5,6,7,7a-hexahydro-4,7-methano-benzo[d] oxazole (19e), Reaction time: $72 \mathrm{~h}$; TLC and purification via column chromatography on silica gel with eluent AcOEt: $\mathrm{CHCl}_{3}: \mathrm{MeOH}, 10: 1: 0.1$; yield: $143 \mathrm{mg}(48 \%)$; white solid; $\operatorname{mp} 112-117^{\circ} \mathrm{C} ;[\alpha]_{\mathrm{D}}^{20}-62.6(\mathrm{c} 1.0, \mathrm{MeOH}) .{ }^{1} \mathrm{H}$ NMR $\left(400 \mathrm{MHz}, \mathrm{CDCl}_{3}\right): \delta=0.90(\mathrm{~s}, 3 \mathrm{H}$, $\left.\mathrm{CH}_{3}\right), 1.07\left(\mathrm{~s}, 3 \mathrm{H}, \mathrm{CH}_{3}\right), 1.11-1.17\left(\mathrm{~m}, 2 \mathrm{H}, \mathrm{CH}_{2}\right), 1.16\left(\mathrm{~s}, 3 \mathrm{H}, \mathrm{CH}_{3}\right), 1.53-1.59(\mathrm{~m}, 1 \mathrm{H}, \mathrm{CH})$, 1.79-11.87 (m, 1H, CH), $2.30(\mathrm{~d}, \mathrm{~J}=4 \mathrm{~Hz}, 1 \mathrm{H}, \mathrm{CH}), 4.39(\mathrm{~d}, \mathrm{~J}=8 \mathrm{~Hz}, 1 \mathrm{H}, \mathrm{CH}), 4.58(\mathrm{~d}$, $\mathrm{J}=8 \mathrm{~Hz}, 1 \mathrm{H}, \mathrm{CH}), 7.64-7.74(\mathrm{~m}, 3 \mathrm{H}, \mathrm{Ar}), 7.80-7.86(\mathrm{~m}, 1 \mathrm{H}, \mathrm{Ar}), 8.64(\mathrm{~d}, \mathrm{~J}=8 \mathrm{~Hz}, 1 \mathrm{H}, \mathrm{Ar})$, $8.22(\mathrm{~d}, \mathrm{~J}=12 \mathrm{~Hz}, 1 \mathrm{H}, \mathrm{Ar}) ;{ }^{13} \mathrm{C}$ NMR $\left(100 \mathrm{MHz}, \mathrm{CDCl}_{3}\right): \delta=11.8,19.4,23.6,26.3,32.3,47.5$, 49.4, 60.5, 91.3, 123.3, 127.1, 127.4, 128.5, 130.4, 130.4, 136.8, 142.0, 164.0; HRMS calculated for $\mathrm{C}_{20} \mathrm{H}_{22} \mathrm{~N}_{2} \mathrm{O}[\mathrm{M}+\mathrm{H}]^{+}$307.1810, found 307.1805.

(S)-1-(4-phenyl-4,5-dihydrooxazol-2-yl)isoquinoline 2-oxide (20c), Reaction time: 5 days; TLC and purification via column chromatography on silica gel with eluent $\mathrm{CH}_{2} \mathrm{Cl}_{2}: \mathrm{MeOH}$, 10:1; yield: $149 \mathrm{mg}$ (58\%); yellowish oil; $[\alpha]_{\mathrm{D}}^{20}-37.5$ (c $\left.0.63, \mathrm{MeOH}\right) .{ }^{1} \mathrm{H}$ NMR $(400 \mathrm{MHz}$, $\left.\mathrm{CDCl}_{3}\right): \delta=4.52(\mathrm{t}, \mathrm{J}=8 \mathrm{~Hz}, 1 \mathrm{H}, \mathrm{N}-\mathrm{CH}), 5.00(\mathrm{dd}, \mathrm{J}=12,8 \mathrm{~Hz}, 1 \mathrm{H}, \mathrm{O}-\mathrm{CH}), 5.62(\mathrm{dd}, \mathrm{J}=12$, $12 \mathrm{~Hz}, 1 \mathrm{H}, \mathrm{O}-\mathrm{CH}), 7.30-7.4(\mathrm{~m}, 1 \mathrm{H}, \mathrm{Ar}), 7.41(\mathrm{t}, \mathrm{J}=8 \mathrm{~Hz}, 2 \mathrm{H}, \mathrm{Ar}), 7.49-7.51(\mathrm{~m}, 2 \mathrm{H}, \mathrm{Ar})$, 7.55-7.65 (m, 2H, Ar), $7.72(\mathrm{~d}, \mathrm{~J}=8 \mathrm{~Hz}, 1 \mathrm{H}, \mathrm{Ar}), 7.78(\mathrm{~d}, \mathrm{~J}=4 \mathrm{~Hz}, 1 \mathrm{H}, \mathrm{Ar}), 7.90(\mathrm{~d}, \mathrm{~J}=8 \mathrm{~Hz}$, $1 \mathrm{H}, \mathrm{Ar}), 8.18(\mathrm{~d}, \mathrm{~J}=8 \mathrm{~Hz}, 1 \mathrm{H}, \mathrm{Ar}) ;{ }^{13} \mathrm{C}$ NMR $\left(100 \mathrm{MHz}, \mathrm{CDCl}_{3}\right): \delta=70.7,75.5,123.9,125.4$, 127.1, 127.1, 128.0, 128.4, 128.8, 128.9, 129.0, 130.2, 137.2, 141.4, 157.9; HRMS calculated for $\mathrm{C}_{18} \mathrm{H}_{14} \mathrm{~N}_{2} \mathrm{O}_{2}[\mathrm{M}+\mathrm{H}]^{+}$291.1133, found 291.1133.

1-((3aR,4S,7R,7aS)-7,8,8-trimethyl-3a,4,5,6,7,7a-hexahydro-4,7-methanobenzo[d]oxazol-2-yl)isoquinoline 2-oxide (20e), Reaction time: $96 \mathrm{~h}$; TLC and purification via column chromatography on silica gel with eluent $\mathrm{CHCl}_{3}: \mathrm{MeOH}, 12: 1$; yield: $13 \mathrm{mg}(10 \%)$; yellow oil; $[\alpha]_{\mathrm{D}}^{20}+$ 21.1 (c 1.0, MeOH). ${ }^{1} \mathrm{H}$ NMR (400 MHz, $\left.\mathrm{CDCl}_{3}\right): \delta=0.91\left(\mathrm{~s}, 3 \mathrm{H}, \mathrm{CH}_{3}\right), 1.10\left(\mathrm{~s}, 3 \mathrm{H}, \mathrm{CH}_{3}\right)$, 1.07-1.15 (m, 2H, $\left.\mathrm{CH}_{2}\right), 1.28\left(\mathrm{~s}, 3 \mathrm{H}, \mathrm{CH}_{3}\right), 1.53-1.59(\mathrm{~m}, 1 \mathrm{H}, \mathrm{CH}), 1.79-1.87(\mathrm{~m}, 1 \mathrm{H}, \mathrm{CH})$, $2.30(\mathrm{~d}, \mathrm{~J}=4 \mathrm{~Hz}, 1 \mathrm{H}, \mathrm{CH}), 4.35(\mathrm{~d}, \mathrm{~J}=8 \mathrm{~Hz}, 1 \mathrm{H}, \mathrm{CH}), 4.64(\mathrm{~d}, \mathrm{~J}=8 \mathrm{~Hz}, 1 \mathrm{H}, \mathrm{CH}), 7.52-7.60$ $(\mathrm{m}, 2 \mathrm{H}, \mathrm{Ar}), 7.64(\mathrm{~d}, \mathrm{~J}=8 \mathrm{~Hz}, 1 \mathrm{H}, \mathrm{Ar}), 7.73(\mathrm{~d}, \mathrm{~J}=8 \mathrm{~Hz}, 1 \mathrm{H}, \mathrm{Ar}), 8.08(\mathrm{~d}, \mathrm{~J}=8 \mathrm{~Hz}, 1 \mathrm{H}$, Ar), $8.13(\mathrm{~d}, \mathrm{~J}=8 \mathrm{~Hz}, 1 \mathrm{H}, \mathrm{Ar}) ;{ }^{13} \mathrm{C} \mathrm{NMR}\left(100 \mathrm{MHz}, \mathrm{CDCl}_{3}\right): \delta=11.6,20.2,23.5,26.6,32.3$, 47.6, 49.0, 49.2, 76.9, 92.4, 124.4, 125.3, 127.1, 128.4, 128.6, 128.9, 130.0, 137.5, 158.7; HRMS calculated for $\mathrm{C}_{20} \mathrm{H}_{22} \mathrm{~N}_{2} \mathrm{O}_{2}[\mathrm{M}+\mathrm{H}]^{+} 323.1760$, found 323.1764.

(S)-2-(4-isopropyl-4,5-dihydrooxazol-2-yl)-3,5-dimethylpyridine 1-oxide (10a), Reaction time: $96 \mathrm{~h}$; TLC and purification via column chromatography on silica gel with eluent $\mathrm{CH}_{2} \mathrm{Cl}_{2}$ : $\mathrm{MeOH}, 10: 1$; yield: $30 \mathrm{mg}$ (19\%); orange gummy liquid; $[\alpha]_{\mathrm{D}}^{20}-39.3$ (c 1.0, $\mathrm{CHCl}_{3}$ ). ${ }^{1} \mathrm{H} \mathrm{NMR}\left(400 \mathrm{MHz}, \mathrm{CDCl}_{3}\right): \delta=0.98\left(\mathrm{~d}, \mathrm{~J}=8 \mathrm{~Hz}, 3 \mathrm{H}, \mathrm{CH}_{3}\right), 1.03\left(\mathrm{~d}, \mathrm{~J}=8 \mathrm{~Hz}, 3 \mathrm{H}, \mathrm{CH}_{3}\right)$, 
1.87-1.92 (m, 1H, $\left.\mathrm{CH}\left(\mathrm{CH}_{3}\right)_{2}\right), 2.25\left(\mathrm{~s}, 3 \mathrm{H}, \mathrm{CH}_{3}\right), 2.29\left(\mathrm{~s}, 3 \mathrm{H}, \mathrm{CH}_{3}\right), 4.15-4.22\left(\mathrm{~m}, 2 \mathrm{H}, \mathrm{O}-\mathrm{CH}_{2}\right)$, 4.48-4.54 (m, 1H, N-CH), 6.94 (s, 1H, Py), 7.97 (s, 1H, Py); ${ }^{13} \mathrm{C}$ NMR (100 MHz, CDCl 3 ): $\delta=18.3,18.7,19.0,32.7,70.9,73.1,128.8,132.4,136.4,136.8,137.3,157.1 ;$ HRMS calculated for $\mathrm{C}_{13} \mathrm{H}_{18} \mathrm{~N}_{2} \mathrm{O}_{2}[\mathrm{M}+\mathrm{H}]^{+}$235.1447, found 235.1453.

(S)-2-(4-(tert-butyl)-4,5-dihydrooxazol-2-yl)-3,5-dimethylpyridine 1-oxide (10b), Reaction time: 5 days; TLC and purification via column chromatography on silica gel with eluent $\mathrm{CH}_{2} \mathrm{Cl}_{2}$ : $\mathrm{MeOH}, 10: 1$; yield: $115 \mathrm{mg}(42 \%)$; yellow oil; $[\alpha]_{\mathrm{D}}^{20}-49.2$ (c 1.34, $\left.\mathrm{CHCl}_{3}\right) .{ }^{1} \mathrm{H}$ NMR

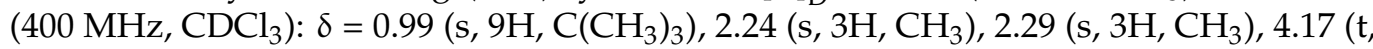
$\mathrm{J}=8 \mathrm{~Hz}, 1 \mathrm{H}, \mathrm{CH}), 4.25(\mathrm{t}, \mathrm{J}=8 \mathrm{~Hz}, 1 \mathrm{H}, \mathrm{CH}), 4.46(\mathrm{t}, \mathrm{J}=8 \mathrm{~Hz}, 1 \mathrm{H}, \mathrm{CH}), 6.92(\mathrm{~s}, 1 \mathrm{H}, \mathrm{Py})$, $7.94(\mathrm{~s}, 1 \mathrm{H}, \mathrm{Py}) ;{ }^{13} \mathrm{C}$ NMR $\left(100 \mathrm{MHz}, \mathrm{CDCl}_{3}\right): \delta=18.4,18.4,26.2,33.9,69.3,76.7,128.5,136.3$, 136.7, 137.3, 157.1; HRMS calculated for $\mathrm{C}_{14} \mathrm{H}_{20} \mathrm{~N}_{2} \mathrm{O}_{2}[\mathrm{M}+\mathrm{H}]^{+} 249.1603$, found 249.1606.

(S)-3,5-dimethyl-2-(4-phenyl-4,5-dihydrooxazol-2-yl)pyridine 1-oxide (10c), Reaction time: $72 \mathrm{~h}$; TLC and purification via column chromatography on silica gel with eluent $\mathrm{CH}_{2} \mathrm{Cl}_{2}: \mathrm{MeOH}$, 10:1; yield: $150 \mathrm{mg}(49 \%)$; yellowish gummy liquid; $[\alpha]_{\mathrm{D}}^{20}-68.2\left(\mathrm{c} 1.0, \mathrm{CHCl}_{3}\right) .{ }^{1} \mathrm{H} \mathrm{NMR}$ $\left(400 \mathrm{MHz}, \mathrm{CDCl}_{3}\right): \delta=2.27\left(\mathrm{~s}, 3 \mathrm{H}, \mathrm{CH}_{3}\right), 2.35\left(\mathrm{~s}, 3 \mathrm{H}, \mathrm{CH}_{3}\right), 4.37(\mathrm{t}, \mathrm{J}=8 \mathrm{~Hz}, 1 \mathrm{H}, \mathrm{N}-\mathrm{CH})$, $4.87(\mathrm{dd}, \mathrm{J}=12,8 \mathrm{~Hz}, 1 \mathrm{H}, \mathrm{O}-\mathrm{CH}), 5.47(\mathrm{dd}, \mathrm{J}=12,8 \mathrm{~Hz}, 1 \mathrm{H}, \mathrm{O}-\mathrm{CH}), 6.95$ (s, 1H, Py), 7.26-7.31 (m, 1H, Ar), 7.34-7.43 (m, 4H, Ar), $7.99(\mathrm{~s}, 1 \mathrm{H}, \mathrm{Py}) ;{ }^{13} \mathrm{C}$ NMR $\left(100 \mathrm{MHz}, \mathrm{CDCl}_{3}\right)$ : $\delta=18.4,18.4,70.4,75.2,127.0,127.9,128.5,128.9,136.4,136.9,137.4,141.6,158.5 ;$ HRMS calculated for $\mathrm{C}_{16} \mathrm{H}_{16} \mathrm{~N}_{2} \mathrm{O}_{2}[\mathrm{M}+\mathrm{H}]^{+}$269.1290, found 269.1286.

2-((3aR,8aS)-3a,8a-dihydro-8H-indeno[1,2-d]oxazol-2-yl)-3,5-dimethylpyridine 1-oxide (10d), Reaction time: $6 \mathrm{~d}$; TLC and purification via column chromatography on silica gel with eluent $\mathrm{CHCl}_{3}: \mathrm{MeOH}, 12: 1$; yield: $132 \mathrm{mg}(42 \%)$; brown, gummy liquid; $[\alpha]_{\mathrm{D}}^{20}+236.0\left(\mathrm{c} 0.5, \mathrm{CHCl}_{3}\right)$. ${ }^{1} \mathrm{H}$ NMR $\left(400 \mathrm{MHz}, \mathrm{CDCl}_{3}\right): \delta=1.95\left(\mathrm{~s}, 3 \mathrm{H}, \mathrm{CH}_{3}\right), 2.23\left(\mathrm{~s}, 3 \mathrm{H}, \mathrm{CH}_{3}\right), 3.36-3.52(\mathrm{~m}, 2 \mathrm{H}$, $\left.\mathrm{CH}_{2}\right), 5.60\left(\mathrm{t}, \mathrm{J}=8 \mathrm{~Hz}, 1 \mathrm{H}, \mathrm{CH}-\mathrm{CH}_{2}\right), 5.80(\mathrm{~d}, \mathrm{~J}=8 \mathrm{~Hz}, 1 \mathrm{H}, \mathrm{CH}), 6.85(\mathrm{~s}, 1 \mathrm{H}, \mathrm{Py}), 7.22-7.29$ $(\mathrm{m}, 4 \mathrm{H}, \mathrm{Ar}), 7.49-7.51(\mathrm{~m}, 1 \mathrm{H}, \mathrm{Ar}), 7.93(\mathrm{~s}, 1 \mathrm{H}, \mathrm{Py}) ;{ }^{13} \mathrm{C} \mathrm{NMR}\left(100 \mathrm{MHz}, \mathrm{CDCl}_{3}\right): \delta=17.8$, 18.3, 39.6, 76.9, 84.0, 125.4, 125.6, 127.6, 128.4, 128.7, 136.7, 136.7, 137.2, 139.7, 141.4, 157.6; HRMS calculated for $\mathrm{C}_{17} \mathrm{H}_{16} \mathrm{~N}_{2} \mathrm{O}_{2}[\mathrm{M}+\mathrm{H}]^{+}$281.1290, found 281.1288.

\subsection{General Procedure for the Oxidation with $m-C P B A$}

Method A: According to the literature [37]: to a solution of a substrate $(0.889 \mathrm{mmol}$, 1 eq.) in dichloromethane $(4 \mathrm{~mL})$, solution of $m$-CPBA $(70 \%, 0.223 \mathrm{~g}, 1$ eq.) in dichloromethane $(6.8 \mathrm{~mL})$ was added dropwise. After complete addition, the reaction mixture was stirred at room temperature for $48 \mathrm{~h}$. The solution was then washed with $\mathrm{NaHCO}_{3}(5 \mathrm{~mL})$, the water phase was extracted with chloroform $(3 \times 7 \mathrm{~mL})$, and the combined organic layers were dried with $\mathrm{MgSO}_{4}$. The solvent was evaporated giving the crude product, which was purified by column chromatography on silica gel.

Method B: According to the literature [38]: to a solution of a substrate (11.1 $\mathrm{mmol}, 1 \mathrm{eq}$.) in dichloromethane $(13.5 \mathrm{~mL})$, solution of $m$-CPBA $(70 \%, 3 \mathrm{~g}, 1.1 \mathrm{eq}$.$) in dichloromethane$ $(8.3 \mathrm{~mL})$ was added dropwise at $0{ }^{\circ} \mathrm{C}$. After complete addition, the reaction was allowed to reach room temperature and was stirred for $24 \mathrm{~h}$. The solution was washed with $\mathrm{NaHCO}_{3}$ $(10 \mathrm{~mL})$, the water phase was extracted with chloroform $(3 \times 15 \mathrm{~mL})$, and the combined organic layers were dried with $\mathrm{MgSO}_{4}$. The solvent was evaporated to give a crude product.

(S)-1-(4-isopropyl-4,5-dihydrooxazol-2-yl)isoquinoline 2-oxide (20a), Method A; purification via column chromatography on silica gel with eluent $\mathrm{CHCl}_{3}: \mathrm{MeOH}, 12: 1$; yield: $15 \mathrm{mg}(14 \%)$; yellow, gummy liquid; $[\alpha]_{\mathrm{D}}^{20}-33.2(\mathrm{c} 0.5, \mathrm{MeOH}) .{ }^{1} \mathrm{H} \mathrm{NMR}\left(400 \mathrm{MHz}, \mathrm{CDCl}_{3}\right): \delta=1.06(\mathrm{~d}$, $\left.\mathrm{J}=8 \mathrm{~Hz}, 3 \mathrm{H}, \mathrm{CH}_{3}\right), 1.13\left(\mathrm{~d}, \mathrm{~J}=8 \mathrm{~Hz}, 3 \mathrm{H}, \mathrm{CH}_{3}\right), 1.97-2.01\left(\mathrm{~m}, 1 \mathrm{H}, \mathrm{CH}\left(\mathrm{CH}_{3}\right)_{2}\right), 4.30-4.35(\mathrm{~m}$, $\left.2 \mathrm{H}, \mathrm{O}-\mathrm{CH}_{2}\right), 4.64(\mathrm{t}, \mathrm{J}=12 \mathrm{~Hz}, 1 \mathrm{H}, \mathrm{N}-\mathrm{CH}), 7.54-7.64(\mathrm{~m}, 2 \mathrm{H}, \mathrm{Ar}), 7.68(\mathrm{~d}, \mathrm{~J}=8 \mathrm{~Hz}, 1 \mathrm{H}, \mathrm{Ar})$ $7.76(\mathrm{~d}, \mathrm{~J}=8 \mathrm{~Hz}, 1 \mathrm{H}, \mathrm{Ar}), 7.82(\mathrm{~d}, \mathrm{~J}=12 \mathrm{~Hz}, 1 \mathrm{H}, \mathrm{Ar}), 8.14(\mathrm{~d}, \mathrm{~J}=8 \mathrm{~Hz}, 1 \mathrm{H}, \mathrm{Ar}) ;{ }^{13} \mathrm{C}$ NMR $\left(100 \mathrm{MHz}, \mathrm{CDCl}_{3}\right): \delta=18.8,19.2,32.9,71.3,73.5,124.0,125.2,126.9,127.0,128.4,128.8,130.1$, 137.1, 140.3, 156.5; HRMS calculated for $\mathrm{C}_{15} \mathrm{H}_{16} \mathrm{~N}_{2} \mathrm{O}_{2}[\mathrm{M}+\mathrm{H}]^{+}$257.1290, found 257.1292.

(S)-1-(4-(tert-butyl)-4,5-dihydrooxazol-2-yl)isoquinoline 2-oxide (20b), Method A; purification via column chromatography on silica gel firstly, with eluent $\mathrm{AcOEt}: \mathrm{CHCl}_{3}: \mathrm{MeOH}, 10: 1: 0.1$ 
to separate contamination, then $\mathrm{CHCl}_{3}: \mathrm{MeOH}, 12: 1$; yield: $138 \mathrm{mg}(58 \%)$; yellow-orange, gummy liquid; $[\alpha]_{\mathrm{D}}^{20}-49.6(\mathrm{c} 0.5, \mathrm{MeOH}) .{ }^{1} \mathrm{H} \mathrm{NMR}\left(400 \mathrm{MHz}, \mathrm{CDCl}_{3}\right): \delta=1.07(\mathrm{~s}, 9 \mathrm{H}$, $\left.\mathrm{C}\left(\mathrm{CH}_{3}\right)_{3}\right), 4.32(\mathrm{dd}, \mathrm{J}=12,8 \mathrm{~Hz} .1 \mathrm{H}, \mathrm{O}-\mathrm{CH}), 4.41(\mathrm{t}, \mathrm{J}=12 \mathrm{~Hz}, 1 \mathrm{H}, \mathrm{N}-\mathrm{CH}), 4.59$ (dd, J = 8, $8 \mathrm{~Hz}, 1 \mathrm{H}, \mathrm{O}-\mathrm{CH}), 7.54-7.64(\mathrm{~m}, 2 \mathrm{H}, \mathrm{Ar}), 7.68$ (d, J = $8 \mathrm{~Hz}, 1 \mathrm{H}, \mathrm{Ar}) 7.76$ (d, J = $8 \mathrm{~Hz}, 1 \mathrm{H}$, Ar), $7.82(\mathrm{~d}, \mathrm{~J}=12 \mathrm{~Hz}, 1 \mathrm{H}, \mathrm{Ar}), 8.14(\mathrm{~d}, \mathrm{~J}=8 \mathrm{~Hz}, 1 \mathrm{H}, \mathrm{Ar}) ;{ }^{13} \mathrm{C}$ NMR $\left(100 \mathrm{MHz}, \mathrm{CDCl}_{3}\right)$ : $\delta=14.3,26.3,34.0,69.7,77.1,124.0,125.1,127.0,128.4,128.8,128.8,130.1,137.2,156.6$; HRMS calculated for $\mathrm{C}_{16} \mathrm{H}_{18} \mathrm{~N}_{2} \mathrm{O}_{2}[\mathrm{M}+\mathrm{H}]^{+} 271.1447$, found 271.1452.

1-((3aR,8aS)-3a,8a-dihydro-8H-indeno[1,2-d]oxazol-2-yl)isoquinoline 2-oxide (20d), Method A; purification via column chromatography on silica gel with eluent $\mathrm{CHCl}_{3}: \mathrm{MeOH}, 10: 1$; yield: $102 \mathrm{mg}(80 \%)$; yellow, gummy liquid; $[\alpha]_{\mathrm{D}}^{20}+115.2$ (c 1.0, MeOH). ${ }^{1} \mathrm{H}$ NMR $(400 \mathrm{MHz}$, $\left.\mathrm{CDCl}_{3}\right): \delta=3.46-3.59\left(\mathrm{~m}, 2 \mathrm{H}, \mathrm{CH}_{2}\right), 5.71-5.75(\mathrm{~m}, 1 \mathrm{H}, \mathrm{CH}), 5.93(\mathrm{~d}, \mathrm{~J}=8 \mathrm{~Hz}, 1 \mathrm{H}, \mathrm{CH})$, 7.31-7.34 (m, 3H, Ar), 7.37-7.44 (m, 2H, Ar) 7.47-7.51 (m, 1H, Ar), 7.58 (t, J = 4 Hz, 1H, Ar), $7.66(\mathrm{~d}, \mathrm{~J}=8 \mathrm{~Hz}, 1 \mathrm{H}, \mathrm{Ar}), 7.72(\mathrm{~d}, \mathrm{~J}=8 \mathrm{~Hz}, 1 \mathrm{H}, \mathrm{Ar}), 8.12(\mathrm{~d}, \mathrm{~J}=4 \mathrm{~Hz}, 1 \mathrm{H}, \mathrm{Ar}) ;{ }^{13} \mathrm{C}$ NMR $\left(100 \mathrm{MHz}_{\mathrm{CDCl}}\right): \delta=39.7,77.2,84.5,123.8,125.3,125.6,125.7,126.9,127.7,128.4,128.8$, $128.9,130.2,137.0,139.8,141.3,157.0$; HRMS calculated for $\mathrm{C}_{19} \mathrm{H}_{14} \mathrm{~N}_{2} \mathrm{O}_{2}[\mathrm{M}+\mathrm{H}]^{+} 303.1133$, found 303.1125 .

3,5-dimethylpyridine 1-oxide (7), Method B; recrystallization from tert-butyl methyl ether; yield: $1.32 \mathrm{~g}$ (77\%); dark orange crystals; ${ }^{1} \mathrm{H}$ NMR $\left(400 \mathrm{MHz}, \mathrm{CDCl}_{3}\right): \delta=2.28(\mathrm{~s}, 6 \mathrm{H})$, $6.90(\mathrm{~s}, 1 \mathrm{H}), 7.88(\mathrm{~s}, 2 \mathrm{H})$; spectroscopic data in agreement with literature data [49].

2-cyano-3,5-dimethylpyridine 1-oxide (9), Method A; purification via recrystallization with methyl tert-butyl ether and then column chromatography on silica gel with eluent $\mathrm{CHCl}_{3}$ : $\mathrm{MeOH}, 12: 1$; yield: 0,462 g (64\%); white needles; mp 137-139 ${ }^{\circ} \mathrm{C} .{ }^{1} \mathrm{H} \mathrm{NMR}(400 \mathrm{MHz}$, $\left.\mathrm{CDCl}_{3}\right): \delta=2.31\left(\mathrm{~s}, 3 \mathrm{H}, \mathrm{CH}_{3}\right), 2.46\left(\mathrm{~s}, 3 \mathrm{H}, \mathrm{CH}_{3}\right), 6.98(\mathrm{~s}, 1 \mathrm{H}, \mathrm{Py}), 7.97(\mathrm{~s}, 1 \mathrm{H}, \mathrm{Py}) ;{ }^{13} \mathrm{C}$ NMR $\left(100 \mathrm{MHz}_{\mathrm{CDCl}}\right): \delta=18.8,19.0,111.4,127.7,137.6,139.7,141.5,149.2$. HRMS calculated for $\mathrm{C}_{8} \mathrm{H}_{8} \mathrm{~N}_{2} \mathrm{O}[\mathrm{M}+\mathrm{H}]^{+}$149.0715, found 149.0716 .

2-(tert-butyl)pyridine 1-oxide (12). Method B; yield: $1.5 \mathrm{~g}(89 \%)$ orange liquid; ${ }^{1} \mathrm{H}$ NMR $\left(400 \mathrm{MHz} \mathrm{CDCl}_{3}\right): \delta=1.49\left(\mathrm{~s}, 9 \mathrm{H}, \mathrm{C}\left(\mathrm{CH}_{3}\right)_{3}\right), 7.08(\mathrm{td}, \mathrm{J}=8,4 \mathrm{~Hz}, 1 \mathrm{H}, \mathrm{Py}), 7.17$ (td, J = 8, $4 \mathrm{~Hz}, 1 \mathrm{H}, \mathrm{Py}), 7.30$ (dd, J = 8, 4 Hz, 1H, Py), 8.16 (dd, J = 8, 4 Hz, 1H, Py); spectroscopic data consistent with literature values [38].

\subsection{General Procedure for Catalytic Allylation of Aromatic Aldehydes with Allyltrichlorosilane}

Based on the report by Chai et al. [50]: a dried Shlenk flask was charged with DIPEA $(0.525 \mathrm{~mL}, 3.0 \mathrm{mmol})$, ligand $(0.009 \mathrm{mmol})$, and aldehyde $(0.60 \mathrm{mmol})$ in $\mathrm{CH}_{3} \mathrm{CN}(3.0 \mathrm{~mL})$ under argon at room temperature. The mixture was cooled to $-40{ }^{\circ} \mathrm{C}$, ally-trichlorosilane $(0.120 \mathrm{~mL}, 0.705 \mathrm{mmol})$ was added and the resulting mixture was stirred at this temperature for $6 \mathrm{~h}$. After that time, the reaction was quenched with aqueous saturated $\mathrm{NaHCO}_{3}$ $(7.5 \mathrm{~mL})$, and the aqueous layer was extracted with ethyl acetate $(3 \times 7.5 \mathrm{~mL})$. The combined organic layers were dried over anhydrous $\mathrm{MgSO}_{4}$. The solvent was removed and purification by flash chromatography on silica gel furnished the known product, for which the spectral data, shown below, are in agreement with the literature values $[39,51]$ :

(S)-1-Phenylbut-3-en-1-ol, Eluent (petroleum ether/ethyl acetate, 95:5, ${ }^{1} \mathrm{H}$ NMR (400 MHz, $\left.\mathrm{CDCl}_{3}\right): \delta=2.05(\mathrm{br}, \mathrm{s}, 1 \mathrm{H}, \mathrm{OH}), 2.45-2.57\left(\mathrm{~m}, 2 \mathrm{H}, \mathrm{CH}_{2}\right), 4.71-4.75(\mathrm{~m}, 1 \mathrm{H},-\mathrm{CH}-\mathrm{OH})$, 5.12-5.19 (m, 2H, =CH $)$, 5.77-5.86 (m, 1H, $\mathrm{CH}=), 7.26-7.32(\mathrm{~m}, 1 \mathrm{H}, \mathrm{Ar}), 7.34-7.37(\mathrm{~m}$, $4 \mathrm{H}, \mathrm{Ar}$ ); chiral HPLC (Chiralpak AS-H, $n$-heksan/ $i$-PrOH, 99:1, $0.6 \mathrm{~mL} / \mathrm{min}$ ), $\lambda=220 \mathrm{~nm}$, $\mathrm{t}_{\mathrm{R}}=30.3 \mathrm{~min}, \mathrm{t}_{\mathrm{S}}=33.4 \mathrm{~min}$ (major)).

(S)-1-(4-Nitrophenyl)but-3-en-1-ol, Eluent (hexane/ethyl acetate, 3:1, ${ }^{1} \mathrm{H}$ NMR (400 MHz, $\left.\mathrm{CDCl}_{3}\right) \delta 2.50-2.42\left(\mathrm{~m}, \mathrm{CH}_{2}\right), 2.48(\mathrm{~s}, 1 \mathrm{H}, \mathrm{OH}), 2.59-2.53\left(\mathrm{~m}, 1 \mathrm{H}, \mathrm{CH}_{2}\right), 4.86(\mathrm{dd}, 1 \mathrm{H}, \mathrm{J}=4$, $8 \mathrm{~Hz},-\mathrm{CH}-\mathrm{OH}), 5.20-5.15\left(\mathrm{~m}, 2 \mathrm{H},=\mathrm{CH}_{2}\right), 5.84-5.73(\mathrm{~m}, 1 \mathrm{H},-\mathrm{CH}=), 7.53(\mathrm{~d}, 2 \mathrm{H}, \mathrm{J}=9.0$ $\mathrm{Hz}, \mathrm{Ar}), 8.19$ (d, 2H, J = $8 \mathrm{~Hz}, \mathrm{Ar}$ ); chiral HPLC (Chiralcel OB-H, $n$-hexane $/ i$-PrOH $=20 / 1$, $0.5 \mathrm{~mL} / \mathrm{min}$ ), $\lambda=254 \mathrm{~nm} \mathrm{t}_{\mathrm{S}}=42.0 \mathrm{~min}$ (major), $\mathrm{t}_{\mathrm{R}}=46.9 \mathrm{~min}$. 
(S)-1-(Naphthalen-1-yl)but-3-en-1-ol, Eluent (hexane/ethyl acetate, 3:1, ${ }^{1} \mathrm{H}$ NMR (400 MHz, $\left.\mathrm{CDCl}_{3}\right) \delta 2.29(\mathrm{~s}, 1 \mathrm{H}, \mathrm{OH}), 2.61-2.53\left(\mathrm{~m}, 1 \mathrm{H}, \mathrm{CH}_{2}\right), 2.77-2.70\left(\mathrm{~m}, 1 \mathrm{H}, \mathrm{CH}_{2}\right), 5.22-5.14(\mathrm{~m}$, $\left.2 \mathrm{H},=\mathrm{CH}_{2}\right), 5.48(\mathrm{q}, 1 \mathrm{H}, \mathrm{J}=4 \mathrm{~Hz},-\mathrm{CH}-\mathrm{OH}), 5.95-5.84(\mathrm{~m}, 1 \mathrm{H},-\mathrm{CH}=), 7.52-7.41(\mathrm{~m}, 3 \mathrm{H}, \mathrm{Ar})$, $7.63(\mathrm{~d}, 1 \mathrm{H}, \mathrm{J}=8 \mathrm{~Hz}, \mathrm{Ar}), 7.76(\mathrm{~d}, 1 \mathrm{H}, \mathrm{J}=8 \mathrm{~Hz}, \mathrm{Ar}), 7.88-7.83(\mathrm{~m}, 1 \mathrm{H}, \mathrm{Ar}), 8.06-8.01(\mathrm{~m}, 1 \mathrm{H}$, Ar); chiral HPLC (Chiralcel OD-H, $n$-hexane $/ i-\mathrm{PrOH}=95 / 5,0.8 \mathrm{~mL} / \mathrm{min}$ ), $\lambda=254 \mathrm{~nm}$, $\mathrm{t}_{\mathrm{S}}=13.9 \mathrm{~min}$ (major), $\mathrm{t}_{\mathrm{R}}=25.3 \mathrm{~min}$.

(S)-1-(Naphthalen-2-yl)but-3-en-1-ol, Eluent (hexane/ethyl acetate, 3:1, ${ }^{1} \mathrm{H}$ NMR $(400 \mathrm{MHz}$, $\left.\mathrm{CDCl}_{3}\right): \delta 2.25(\mathrm{br} \mathrm{s}, 1 \mathrm{H}, \mathrm{OH}), 2.62-2.52\left(\mathrm{~m}, 2 \mathrm{H}, \mathrm{CH}_{2}\right), 4.87(\mathrm{dd}, \mathrm{J}=4,8 \mathrm{~Hz}, 1 \mathrm{H},-\mathrm{CH}-\mathrm{OH})$, 5.19-5.12 $\left(\mathrm{m}, 2 \mathrm{H},=\mathrm{CH}_{2}\right), 5.86-5.76(\mathrm{~m}, 1 \mathrm{H},-\mathrm{CH}=), 7.49-7.43(\mathrm{~m}, 3 \mathrm{H}, \mathrm{Ar}), 7.82-7.78(\mathrm{~m}$, $4 \mathrm{H}, \mathrm{Ar}$ ); chiral HPLC (Chiralcel OD-H, $n$-hexane $/ i-\mathrm{PrOH}=9 / 1,0.75 \mathrm{~mL} / \mathrm{min}$ ), $\lambda=254 \mathrm{~nm}$, $\mathrm{t}_{\mathrm{S}}=12.6 \mathrm{~min}$ (major), $\mathrm{t}_{\mathrm{R}}=14.2 \mathrm{~min}$.

\subsection{General Procedure for Catalytic Nitroaldol Reaction}

The solution of $\mathrm{Cu}(\mathrm{OAc})_{2} \cdot \mathrm{H}_{2} \mathrm{O}(10 \mathrm{mg}, 0.05 \mathrm{mmol}, 10 \% \mathrm{~mol})$ and ligand $(0.06 \mathrm{mmol}$, $12 \% \mathrm{~mol}$ ) in $1 \mathrm{~mL}$ of isopropanol was stirred at room temperature for one hour. After this time, benzaldehyde $(0.051 \mathrm{~mL}, 0.5 \mathrm{mmol}, 1.0$ eq.) and an additional $1 \mathrm{~mL}$ of $i-\mathrm{PrOH}$ were added. The reaction mixture was cooled in an ice-water bath to reach $0{ }^{\circ} \mathrm{C}$, and nitromethane $(0.270 \mathrm{~mL}, 5.0 \mathrm{mmol}, 10 \mathrm{eq}$. $)$ was then added. The resulting mixture was stirred for 3 or 6 days at $0-5^{\circ} \mathrm{C}$. Then, the reaction mixture was filtered through silica gel, washed with chloroform, and after evaporation the obtained product was identified by the ${ }^{1} \mathrm{H}$ NMR [52].

1-phenyl-2-nitroethane-1-ol, ${ }^{1} \mathrm{H}$ NMR $\left(400 \mathrm{MHz}, \mathrm{CDCl}_{3}\right): \delta=2.81(\mathrm{br}, \mathrm{s}, 1 \mathrm{H}, \mathrm{OH}), 4.51(\mathrm{dd}$, $\left.\mathrm{J}=4,8 \mathrm{~Hz}, 1 \mathrm{H},-\mathrm{CH}_{2}-\mathrm{NO}_{2}\right), 4.61\left(\mathrm{dd}, \mathrm{J}=8,16 \mathrm{~Hz}, 1 \mathrm{H},-\mathrm{CH}_{2}-\mathrm{NO}_{2}\right), 5.46(\mathrm{~d}, \mathrm{~J}=8 \mathrm{~Hz}$, $1 \mathrm{H},-\mathrm{CH}-\mathrm{OH}), 7.36-7.42(\mathrm{~m}, 5 \mathrm{H}, \mathrm{Ar})$; spectroscopic data consistent with literature values [52]; chiral HPLC (Chiracel OD-H, $n$-hexane/ $i-\mathrm{PrOH}, 9: 1,1.0 \mathrm{~mL} / \mathrm{min}, \lambda=225 \mathrm{~nm}$, $\left.\mathrm{t}_{\mathrm{R}}=16.5 \mathrm{~min}, \mathrm{t}_{\mathrm{S}}=19.8 \mathrm{~min}\right)$.

\section{Conclusions}

Dependent on building the azaaromatic framework, two different synthetic routes were successfully applied to obtain a series of novel chiral heteroaromatic $\mathrm{N}$-oxide oxazolines, compounds combining unique properties of $\mathrm{N}$-oxide and oxazoline moieties. For the obtaining of pyridine and bipyridine $\mathrm{N}$-oxide based novel oxazolines $(4,5)$, reaction of $\mathrm{N}$-oxide imidate with chiral amino alcohol in presence of molecular sieves (4A) in toluene at elevated temperature was most convenient, while for alkyl-substituted pyridine $\mathrm{N}$-oxide, the same protocol was impossible to carry out, which indicates how differentially behaved are these seemingly similar structures. The direct procedure for the oxazoline ring closure of N-oxide nitrile with chiral amino alcohol was employed for the synthesis of both 3,5dimethylpyridine- and isoquinoline-substituted-oxazoline $\mathrm{N}$-oxides $(\mathbf{1 0}, \mathbf{2 0}$, respectively). In the case also of the preparation of heteroaromatic $\mathrm{N}$-oxides with an imidazoline moiety $(22,26)$, it was necessary to carry out the oxidation at an earlier stage (nitrile), followed by the closure of the imidazole ring with $(1 R, 2 R)$-diamino-cyclohexane and the generated in situ imidate. Oxidation of previously obtained imidazolines, as well as in the case of 2-tert-butylpyridine-6-oxazolines, failed.

The resulting chiral heteroaromatic $\mathrm{N}$-oxides were applied in catalytic allylation of aromatic aldehydes, giving the corresponding allylic alcohols with moderate yields and enantioselectivity up to $79 \%$. The best results were obtained with $\mathrm{N}$-oxides derived from pyridine, 3,5-lutidine, and isoquinoline with a fragment of (S)-phenyl-glycinol as an inducer of chirality. The obtained results indicate a meaningful role of possible $\pi-\pi$ interactions of both aromatic rings, of the catalyst and substrate in the transition state, which enhance the enantioselectivity. Selected oxazoline $\mathrm{N}$-oxide products were used as chiral ligands in copper(II)-complex catalyzed nitroaldol reaction. In this reaction, the results were higher in terms of efficiency but significantly lower in terms of enantioselectivity (up to $48 \%$ ). 
Supplementary Materials: The following are available online at https:/ / www.mdpi.com/article/10 $.3390 /$ catal11040444/s1, Figure S1. ${ }^{1} \mathrm{H}$ and ${ }^{13} \mathrm{C}$ NMR spectra of 2-(imino(methoxy)methyl)pyridine 1-oxide-3, Figure S2. ${ }^{1} \mathrm{H}$ and ${ }^{13} \mathrm{C}$ NMR spectra of $6^{\prime}$-(imino(methoxy)methyl)-[2,2' -bipyridine] 1-oxide, Figure S3. ${ }^{1} \mathrm{H}$ and ${ }^{13} \mathrm{C}$ NMR spectra of (S)-2-(4-isopropyl-4,5-dihydrooxazol-2-yl)pyridine 1oxide-4a, Figure S4. ${ }^{1} \mathrm{H}$ and ${ }^{13} \mathrm{C}$ NMR spectra of (S)-2-(4-(tert-butyl)-4,5-dihydrooxazol-2-yl)pyridine 1-oxide-4b, Figure S5. ${ }^{1} \mathrm{H}$ and ${ }^{13} \mathrm{C}$ NMR spectra of (S)-2-(4-phenyl-4,5-dihydrooxazol-2-yl)pyridine 1-oxide-4c, Figure $S 6 .{ }^{1} \mathrm{H}$ and ${ }^{13} \mathrm{C}$ NMR spectra of 2-((3aR,8aS)-3a,8a-dihydro-8H-indeno[1,2-d]oxazol2-yl)pyridine 1-oxide-4d, Figure S7. ${ }^{1} \mathrm{H}$ and ${ }^{13} \mathrm{C}$ NMR spectra of 2-((3aR,4S,7R,7aS)-7,8,8-trimethyl3a,4,5,6,7,7a-hexahydro-4,7-methanobenzo[d] oxazol-2-yl)pyridine 1-oxide-4e, Figure $S 8 .{ }^{1} \mathrm{H}$ and ${ }^{13} \mathrm{C}$ NMR spectra of (S)-6' -(4-isopropyl-4,5-dihydrooxazol-2-yl)-[2,2' -bipyridine] 1-oxide-5a, Figure S9. ${ }^{1} \mathrm{H}$ and ${ }^{13} \mathrm{C}$ NMR spectra of (S)-6' -(4-(tert-butyl)-4,5-dihydrooxazol-2-yl)-[2,2' -bipyridine] 1-oxide-5b, Figure S10. ${ }^{1} \mathrm{H}$ and ${ }^{13} \mathrm{C}$ NMR spectra of $(S)-6^{\prime}$-(4-phenyl-4,5-dihydrooxazol-2-yl)-[2,2' -bipyridine] 1-oxide-5c, Figure S11. ${ }^{1} \mathrm{H}$ and ${ }^{13} \mathrm{C}$ NMR spectra of $6^{\prime}-((3 \mathrm{a} R, 8 \mathrm{a} S)-3 \mathrm{a}, 8 \mathrm{a}-$ dihydro-8H-indeno[1,2d]oxazol-2-yl)-[2,2' -bipyridine] 1-oxide-5d, Figure S12. ${ }^{1} \mathrm{H}$ and ${ }^{13} \mathrm{C}$ NMR spectra of 2-cyano3,5-dimethylpyridine 1-oxide-9, Figure S13. ${ }^{1} \mathrm{H}$ and ${ }^{13} \mathrm{C}$ NMR spectra of (S)-2-(4-isopropyl-4,5dihydroox-azol-2-yl)-3,5-dimethylpyridine 1-oxide-10a, Figure S14. ${ }^{1} \mathrm{H}$ and ${ }^{13} \mathrm{C}$ NMR spectra of (S)-2-(4-(tert-butyl)-4,5-dihydrooxazol-2-yl)-3,5-dimethylpyridine 1-oxide-10b, Figure S15. ${ }^{1} \mathrm{H}$ and ${ }^{13} \mathrm{C}$ NMR spectra of (S)-3,5-dimethyl-2-(4-phenyl-4,5-dihydrooxazol-2-yl)pyridine 1-oxide-10c, Figure S16. ${ }^{1} \mathrm{H}$ and ${ }^{13} \mathrm{C}$ NMR spectra of 2-((3aR,8aS)-3a,8a-dihydro-8H-indeno[1,2- $d$ ] yl)-3,5-dimethylpyridine 1-oxide-10d, Figure S17. ${ }^{1} \mathrm{H}$ and ${ }^{13} \mathrm{C}$ NMR spectra of methyl 6-(tertbutyl)picolinimidate-14, Figure S18. ${ }^{1} \mathrm{H}$ and ${ }^{13} \mathrm{C}$ NMR spectra of (S)-2-(6-(tert-butyl)pyridin-2yl)-4-phenyl-4,5-dihydrooxazole-15c, Figure S19. ${ }^{1} \mathrm{H}$ and ${ }^{13} \mathrm{C}$ NMR spectra of $(3 \mathrm{aR}, 8 \mathrm{aS})-2-(6-($ tertbutyl)pyridin-2-yl)-3a,8a-dihydro-8H-indeno[1,2- $d$ ] oxazole-15d, Figure S20. ${ }^{1} \mathrm{H}$ and ${ }^{13} \mathrm{C} \mathrm{NMR}$ spectra of (3aR,4S,7R,7aS)-2-(6-(tert-butyl)-pyridin-2-yl)-7,8,8-trimethyl-3a,4,5,6,7,7a-hexahydro-4,7-methanobenzo[ $d$ ] oxazole-15e, Figure S21. ${ }^{1} \mathrm{H}$ and ${ }^{13} \mathrm{C}$ NMR spectra of $(3 \mathrm{a} R, 4 S, 7 R, 7 \mathrm{a} S)$-2-(isoquinolin1-yl)-7,8,8-trimethyl-3a,4,5,6,7,7a-hexahydro-4,7-methanobenzo[d]oxazole-19e, Figure S22. ${ }^{1} \mathrm{H}$ and ${ }^{13}$ C NMR spectra of (S)-1-(4-isopropyl-4,5-dihydrooxazol-2-yl)isoquinoline 2-oxide-20a, Figure S23. ${ }^{1} \mathrm{H}$ and ${ }^{13} \mathrm{C}$ NMR spectra of (S)-1-(4-(tert-butyl)-4,5-dihydrooxazol-2-yl)isoquinoline 2-oxide-20b, Figure S24. ${ }^{1} \mathrm{H}$ and ${ }^{13} \mathrm{C}$ NMR spectra of (S)-1-(4-phenyl-4,5-dihydrooxazol-2-yl)isoquinoline 2oxide-20c, Figure S25. ${ }^{1} \mathrm{H}$ and ${ }^{13} \mathrm{C}$ NMR spectra of $1-((3 \mathrm{a} R, 8 \mathrm{aS})-3 \mathrm{a}, 8 \mathrm{a}$-dihydro- $8 H$-indeno[1,2d] oxazol-2-yl)isoquinoline 2-oxide-20d, Figure S26. ${ }^{1} \mathrm{H}$ and ${ }^{13} \mathrm{C}$ NMR spectra of $1-((3 \mathrm{a} R, 4 S, 7 R, 7 \mathrm{a} S)$ 7,8,8-trimethyl-3a,4,5,6,7,7a-hexahydro-4,7-methanobenzo[d]oxazol-2-yl)isoquinoline 2-oxide-20e, Figure S27. ${ }^{1} \mathrm{H}$ and ${ }^{13} \mathrm{C}$ NMR spectra of $(Z)-N, N^{\prime}$-bis((S)-2-hydroxy-1-phenylethyl)isoquinoline-1carboximidamide-21, Figure S28. ${ }^{1} \mathrm{H}$ and ${ }^{13} \mathrm{C}$ NMR spectra of 2-((3aR,7aR)-3a, 4,5,6,7,7a-hexahydro1H-benzo[d]imidazol-2-yl)pyridine 1-oxide-22, Figure S29. ${ }^{1} \mathrm{H}$ and ${ }^{13} \mathrm{C}$ NMR spectra of $6^{\prime}-((3 \mathrm{a} R, 7 \mathrm{a} R)$ 3a,4,5,6,7,7a-hexahydro-1H-benzo[d]imidazol-2-yl)-[2,2' -bipyridine] 1-oxide-26, Figure S30. HPLC chromatogram for benzaldehyde-racemate (obtained with non-chiral catalyst 2,2' -bipyridine $\mathrm{N}$ oxide), Figure S31. HPLC chromatogram for benzaldehyde: sample obtained with catalyst 4a, Figure S32. HPLC chromatogram for benzaldehyde: sample obtained with catalyst $4 \mathbf{b}$, Figure S33. HPLC chromatogram for benzaldehyde: sample obtained with catalyst 4c, Figure S34. HPLC chromatogram for benzaldehyde: sample obtained with catalyst $4 \mathrm{c}$ in $\mathrm{CH}_{2} \mathrm{Cl}_{2}$, Figure S35. HPLC chromatogram for benzaldehyde: sample obtained with catalyst 4d, Figure S36. HPLC chromatogram for benzaldehyde: sample obtained with catalyst 4e, Figure S37. HPLC chromatogram for benzaldehyde: sample obtained with catalyst 5b, Figure S38. HPLC chromatogram for benzaldehyde: sample obtained with catalyst 5d, Figure S39. HPLC chromatogram for benzaldehyde: sample obtained with catalyst 10a, Figure S40. HPLC chromatogram for benzaldehyde: sample obtained with catalyst 10b, Figure S41. HPLC chromatogram for benzaldehyde: sample obtained with catalyst 10c, Figure S42. HPLC chromatogram for benzaldehyde: sample obtained with catalyst 10d, Figure S43. HPLC chromatogram for benzaldehyde: sample obtained with catalyst 20a, Figure S44. HPLC chromatogram for benzaldehyde: sample obtained with catalyst 20c, Figure S45. HPLC chromatogram for benzaldehyde: sample obtained with catalyst 20d, Figure S46. HPLC chromatogram for benzaldehyde: sample obtained with catalyst 20e, Figure S47. HPLC chromatogram for benzaldehyde: sample obtained with catalyst 22, Figure S48. HPLC chromatogram for $p$-nitro-benzaldehyde: sample obtained with catalyst 4c, Figure S49. HPLC chromatogram for $p$-nitro-benzaldehyde: sample obtained with catalyst 10c, Figure S50. HPLC chromatogram for $p$-nitro-benzaldehyde: sample obtained with catalyst 20c, Figure S51. HPLC chromatogram for 1-naphthaldehyde: sample obtained with catalyst 4c, Figure S52. HPLC chromatogram for 1-naphthaldehyde: sample obtained 
with catalyst 10c, Figure S53. HPLC chromatogram for 1-naphthaldehyde: sample obtained with catalyst 20c, Figure S54. HPLC chromatogram for 2-naphthaldehyde: sample obtained with catalyst 4c, Figure S55. HPLC chromatogram for 2-naphthaldehyde: sample obtained with catalyst 10c, Figure S56. HPLC chromatogram for 2-naphthaldehyde: sample obtained with catalyst 20c, Figure S57. HPLC chromatogram for racemate, Figure S58. HPLC chromatogram for sample obtained with catalyst 4a, Figure S59. HPLC chromatogram for sample obtained with catalyst 4c, Figure S60. HPLC chromatogram for sample obtained with catalyst 4e, Figure S61. HPLC chromatogram for sample obtained with catalyst 22.

Author Contributions: Conceptualization, R.S. and Z.W.; methodology, R.S.; software, R.S.; validation, R.S., Z.W.; formal analysis, R.S.; investigation, Z.W.; resources, R.S., Z.W.; data curation, Z.W.; writing一original draft preparation, R.S., Z.W.; writing-review and editing, R.S.; visualization, R.S., Z.W. supervision, R.S.; project administration, R.S.; funding acquisition, R.S. All authors have read and agreed to the published version of the manuscript.

Funding: This research received no external funding.

Data Availability Statement: The data are available on request from the corresponding author.

Acknowledgments: Authors are grateful for the financial support given by the Polish Ministry of Science and Higher Education by subvention activity for the Faculty of Chemistry at Wrocław University of Science and Technology.

Conflicts of Interest: The authors declare no conflict of interest.

\section{References}

1. Koukal, P.; Ulč, J.; Nečas, D.; Kotora, M. Pyridine N-Oxides and Derivatives Thereof in Organocatalysis. Top. Heterocycl. Chem. 2017, 53, 29-58. [CrossRef]

2. Zhang, L.; Wang, Y. Recent Developments in the Chemistry of Heteroaromatic N-Oxides. Synthesis 2015, 47, 289-305. [CrossRef]

3. Larionov, O.V.; Stephens, D.; Mfuh, A.M.; Arman, H.D.; Naumova, A.S.; Chavez, G.; Skenderi, B. Insights into the mechanistic and synthetic aspects of the Mo/P-catalyzed oxidation of N-heterocycles. Org. Biomol. Chem. 2014, 12, 3026-3036. [CrossRef]

4. Limnios, D.; Kokotos, C.G. 2,2,2-Trifluoroacetophenone as an Organocatalyst for the Oxidation of Tertiary Amines and Azines to N-Oxides. Chem. Eur. J. 2014, 20, 559-563. [CrossRef] [PubMed]

5. Joule, J.A.; Mills, K. Heterocyclic Chemistry, 5th ed.; John Wiley \& Sons, Inc.: Hoboken, NJ, USA, 2010.

6. Malkov, A.V.; Kocovsky, P. Chiral N-Oxides in Asymmetric Catalysis. CheminInform 2007, 38, 29-36. [CrossRef]

7. Bernier, D.; Wefelscheid, U.K.; Woodward, S. ChemInform Abstract: Properties, Preparation and Synthetic Uses of Amine N-Oxides. An Update. CheminInform 2009, 40, 173-210. [CrossRef]

8. Chelucci, G.; Murineddu, G.; A Pinna, G. Chiral pyridine N-oxides: Useful ligands for asymmetric catalysis. Tetrahedron Asymmetry 2004, 15, 1373-1389. [CrossRef]

9. Gómez, M.; Muller, G.; Rocamora, M. Coordination chemistry of oxazoline ligands. Coord. Chem. Rev. 1999, 193-195, 769-835. [CrossRef]

10. McManus, H.A.; Guiry, P.J. Recent Developments in the Application of Oxazoline-Containing Ligands in Asymmetric Catalysis. Chem. Rev. 2004, 104, 4151-4202. [CrossRef] [PubMed]

11. Helmchen, G.; Pfaltz, A. ChemInform Abstract: Phosphinooxazolines-A New Class of Versatile, Modular P,N-Ligands for Asymmetric Catalysis. CheminInform 2010, 31, 336-345. [CrossRef]

12. Hargadenand, G.C.; Guiry, P.J. Recent Applications of Oxazoline-Containing Ligands in Asymmetric Catalysis. Chem. Rev. 2009, 109, 2505-2550. [CrossRef]

13. Meyers, A.I. Chiral Oxazolines and Their Legacy in Asymmetric Carbon-Carbon Bond-Forming Reactions. J. Org. Chem. 2005, 70, 6137-6151. [CrossRef] [PubMed]

14. Chebanov, V.A.; Gura, K.A.; Desenko, S.M. ChemInform Abstract: Aminoazoles as Key Reagents in Multicomponent Heterocyclizations. CheminInform 2011, 42, 41-84. [CrossRef]

15. Bansal, S.; Halve, A.K. ChemInform Abstract: Oxazolines: Their Synthesis and Biological Activity. CheminInform 2015, 46, 4601-4616. [CrossRef]

16. Brunner, H.; Obermann, U.; Wimmer, P. Asymmetrische katalysen: XXXII. Enantioselektive phenylierung von cis-cyclohexan-1,2diol und meso-butan-2,3-diol. J. Organomet. Chem. 1986, 316, C1-C3. [CrossRef]

17. Yang, G.; Zhang, W. Renaissance of pyridine-oxazolines as chiral ligands for asymmetric catalysis. Chem. Soc. Rev. 2018, 47, 1783-1810. [CrossRef]

18. Copéret, C.; Adolfsson, H.; Khuong, T.A.V.; Yudin, A.A.K.; Sharpless, K.B. A Simple and Efficient Method for the Preparation of PyridineN-Oxides. J. Org. Chem. 1998, 63, 1740-1741. [CrossRef]

19. Shaker, Y. Recent trends in the chemistry of pyridine N-oxides. Arkivoc 2001, 1, 242-268. 
20. Tydlitát, J.; Bureš, F.; Kulhánek, J.; Růžička, A. 1,2-Disubstituted Hexahydro-1H-benzo[d]imidazoles: Synthesis, Characterization, and Stability. Synthesis 2010, 22, 3934-3940.

21. Malkov, A.V.; Liddon, A.J.P.S.; Bendová, L.; Haigh, D.; Kočovský, P.; Ramírez-López, P.; Biedermannová, L. Remote Chiral Induction in the Organocatalytic Hydrosilylation of Aromatic Ketones and Ketimines. Angew. Chem. Int. Ed. 2006, 45, 1432-1435. [CrossRef]

22. Chelucci, G.; Medici, S.; Saba, A. Steric effects of the ligand in the enantioselective palladium-catalyzed allylic alkylation using chiral oxazolinylpyridines. Tetrahedron Asymmetry 1999, 10, 543-550. [CrossRef]

23. Emerson, T.R.; Rees, C.W. 360. The deoxygenation of heterocyclic N-oxides. Part I. A novel oxygen- and peroxide-catalysed reduction of pyridine 1-oxide with triethyl phosphite. J. Chem. Soc. 1962, 1917-1923. [CrossRef]

24. Howard, E.; Olszewski, W.F. The Reaction of Triphenylphosphine with Some Aromatic Amine Oxides1. J. Am. Chem. Soc. 1959, 81, 1483-1484. [CrossRef]

25. Wenkert, D.; Woodward, R.B. Studies of 2,2'-bipyridyl N,N'-dioxides. J. Org. Chem. 1983, 48, 283-289. [CrossRef]

26. Singh, S.K.; Reddy, M.S.; Mangle, M.; Ganesh, K.R. Cu(I)-mediated deoxygenation of N-oxides to amines. Tetrahedron 2007, 63, 126-130. [CrossRef]

27. Reis, M.; Royo, B. Chemoselective hydrogenation of nitroarenes and deoxygenation of pyridine $\mathrm{N}$-oxides with $\mathrm{H} 2$ catalyzed by MoO2Cl2. Tetrahedron Lett. 2009, 50, 949-952. [CrossRef]

28. Toganoh, M.; Fujino, K.; Ikeda, S.; Furuta, H. Catalytic deoxygenation of pyridine N-oxides with N-fused porphyrin rhenium complexes. Tetrahedron Lett. 2008, 49, 1488-1491. [CrossRef]

29. Sanz, R.; Escribano, J.; Fernández, Y.; Aguado, R.; Pedrosa, M.R.; Arnáiz, F.J. Deoxygenation of N-Oxides with Triphenylphosphine, Catalyzed by Dichlorodioxomolybdenum(VI). Synlett 2005, 9, 1389-1392. [CrossRef]

30. Clarke, M.; Fuentes, J. Deoxygenation of Pyridine N-Oxides by Palladium-Catalysed Transfer-Oxidation of Trialkylamines. Synlett 2008, 2008, 2579-2582. [CrossRef]

31. Sedenkova, K.N.; Terekhin, A.V.; Abdrashitova, I.V.; Vasilenko, D.A.; Sadovnikov, K.S.; Gracheva, Y.A.; Grishin, Y.K.; Holt, T.; Kutateladze, A.G.; Kuznetsova, T.S.; et al. Dichotomy in the reactivity of 2-methyltetrahydroquinazoline 1-oxides towards aldehydes: An unprecedented condensation with simultaneous reduction of the N-oxide fragment. Tetrahedron Lett. 2020, 61, 151605. [CrossRef]

32. Frauenlob, R.; McCormack, M.M.; Walsh, C.M.; Bergin, E. Rapid, in situ synthesis of bidentate ligands: Chromatography-free generation of catalyst libraries. Org. Biomol. Chem. 2011, 9, 6934-6937. [CrossRef]

33. Periasamy, M.; Sanjeevakumar, N.; Reddy, P.O. Convenient Methods to Access Chiral Camphanyl Amine Derivatives by Sodium Borohydride Reduction of d-(-)-Camphorquinone Imines. Synthesis 2012, 44, 3185-3190. [CrossRef]

34. Lobert, M.; Köhn, U.; Hoogenboom, R.; Schubert, U.S. Synthesis and microwave assisted polymerization of fluorinated 2-phenyl2-oxazolines: The fastest 2-oxazoline monomer to date. Chem. Commun. 2008, 1458-1460. [CrossRef]

35. Chelucci, G.; Sanna, M.G.; Gladiali, S. Enantioselective Cyclopropanation of Styrene Catalysed by Copper(I) Complexes with Chiral Oxazolines. Tetrahedron 2000, 56, 2889-2893. [CrossRef]

36. García, J.I.; Pires, E.; Cornejo, A.; Fraile, J.M.; Gil, M.J.; Martínez-Merino, V.; Mayoral, J.A.; Villalba, I. An Efficient and General One-Pot Method for the Synthesis of Chiral Bis(oxazoline) and Pyridine Bis(oxazoline) Ligands. Synlett 2005, 2005, $2321-2324$. [CrossRef]

37. Gnanamani, E.; Someshwar, N.; Sanjeevi, J.; Ramanathan, C.R. Conformationally Rigid Chiral Bicyclic Skeleton-Tethered Bipyridine N,N'-Dioxide as Organocatalyst: Asymmetric Ring Opening of meso-Epoxides. Adv. Synth. Catal. 2014, 356, $2219-2223$. [CrossRef]

38. Nienkemper, K.; Kotov, V.V.; Kehr, G.; Erker, G.; Fröhlich, R. Chelate [2-(Iminoethyl)pyridineN-oxide]metal Complexes - Synthesis and Structural Comparison with Their Chemically Related 2-(Iminoethyl)pyridine-Derived Systems. Eur. J. Inorg. Chem. 2006, 2006, 366-379. [CrossRef]

39. Sevrain, N.; Volle, J.N.; Pirat, J.L.; Ayad, T.; Virieux, D. Chiral Bisdiphenylphosphine Dioxides Bearing a Bis(triazolyl) Backbone as Promising Lewis Bases for Asymmetric Organocatalysis. Eur. J. Org. Chem. 2018, 2018, 2267-2272. [CrossRef]

40. Malkov, A.V.; Ramírez-López, P.; Bendová, L.; Rulíšek, L.; Dufkova, L.; Kotora, M.; Zhu, F.; Kočovský, P. On the Mechanism of Asymmetric Allylation of Aldehydes with Allyltrichlorosilanes Catalyzed by QUINOX, a Chiral IsoquinolineN-Oxide. J. Am. Chem. Soc. 2008, 130, 5341-5348. [CrossRef]

41. Rassadin, V.A.; Zimin, D.P.; Raskil'Dina, G.Z.; Ivanov, A.Y.; Boyarskiy, V.P.; Zlotskii, S.S.; Kukushkin, V.Y. Solvent- and halide-free synthesis of pyridine-2-yl substituted ureas through facile $\mathrm{C}-\mathrm{H}$ functionalization of pyridine N-oxides. Green Chem. 2016, 18, 6630-6636. [CrossRef]

42. Bullock, S.J.; Harding, L.P.; Moore, M.P.; Mills, A.; Piela, S.A.F.; Rice, C.R.; Towns-Andrews, L.; Whitehead, M.; Towns-Andrews, E. Synthesis of ligands containing N-oxide donor atoms and their assembly into metallosupramolecular structures. Dalton Trans. 2013, 42, 5805-5811. [CrossRef] [PubMed]

43. Kobayashi, Y.; Kumadaki, I.; Sato, H. Studies on the Reaction of Heterocyclic Compounds. VII. Oxidative Cyanation of Heteroaromatic, N.-Oxides. J. Org. Chem. 1972, 37, 3588-3591. [CrossRef]

44. Norrby, T.; Börje, A.; Zhang, L.; Åkermark, B.; Wagenknecht, J.H.; Francis, G.W.; Szunyog, J.; Långström, B. Regioselective Functionalization of 2,2'-Bipyridine and Transformations into Unsymmetric Ligands for Coordination Chemistry. Acta Chem. Scand. 1998, 52, 77-85. [CrossRef] 
45. Katritzky, A.R.; Scriven, E.F.V.; Majumder, S.; Tu, H.; Vakulenko, A.V.; Akhmedov, N.G.; Murugan, R. Preparation of Cyanopyridines by Direct Cyanation. Synthesis 2005, 6, 993-997. [CrossRef]

46. Du, W.; Gu, Q.; Li, Y.; Lin, Z.; Yang, D. Enantioselective Palladium-Catalyzed Oxidative Cascade Cyclization of Aliphatic Alkenyl Amides. Org. Lett. 2017, 19, 316-319. [CrossRef]

47. Pezzetta, C.; Bonifazi, D.; Davidson, R.W.M. Enantioselective Synthesis of N-Benzylic Heterocycles: A Nickel and Photoredox Dual Catalysis Approach. Org. Lett. 2019, 21, 8957-8961. [CrossRef] [PubMed]

48. Huang, W.; Wan, X.; Shen, Q. Enantioselective Construction of Trifluoromethoxylated Stereogenic Centers by a Nick-el-Catalyzed Asymmetric Suzuki-Miyaura Coupling of Secondary Benzyl Bromides. Angew. Chem. Int. Ed. 2017, 56, 11986-11989. [CrossRef] [PubMed]

49. Benz, S.; Nötzli, S.; Siegel, J.S.; Eberli, D.; Jessen, H.J. Controlled Oxygen Release from Pyridone Endoperoxides Promotes Cell Survival under Anoxic Conditions. J. Med. Chem. 2013, 56, 10171-10182. [CrossRef] [PubMed]

50. Chai, Q.; Song, C.; Sun, Z.; Ma, Y.; Ma, C.; Daia, Y.; Andrus, M.B. Asymmetric allylation of aldehydes with allyltri-chlorosilane using aza-paracyclophane-oxazoline-N-oxide catalysts. Tetrahedron Lett. 2006, 47, 8611-8615. [CrossRef]

51. Yanagisawa, A.; Yang, N.; Bamba, K. Asymmetric Allylation of Carbonyl Compounds Catalyzed by a Chiral Phosphine-Silver Complex. Eur. J. Org. Chem. 2017, 2017, 6614-6618. [CrossRef]

52. Kowalczyk, R.; Sidorowicz, Ł.; Skarżewski, J. Asymmetric Henry reaction catalyzed by chiral secondarydiamine-copper(II) complexes. Tetrahedron Asymmetry 2008, 19, 2310-2315. [CrossRef] 